Series A

I. MATHEMATICA

DISSERTATIONES

64

\title{
GAMES AND INFINITARY LANGUAGES
}

\author{
TAPANI HYTTINEN
}

To be presented, with the permission of the Faculty of Science of the

University of Helsinki, for public criticism in Auditorium XII, on February 14th, 1987, at 10 o'clock a.m.

H E L I N K I 1987

SUOM A L A I N E T IE DEAKATEM I A

https://doi.org/10.5186/aasfmd.1987.020 
Copyright ${ }^{\circledR} 1987$ by Academia Scientiarum Fennica

ISSN 0355-0087

ISBN 951-41-0543-5

Received 27 November 1986 


\section{Acknowledgement}

I wish to express my gratitude to all my teachers, especially to my supervisor Docent Juha Oikkonen, to Asst. Prof. Jouko Väänänen, to Dr. Wilfrid Hodges and to Prof. Jaakko Hintikka, who made me a member of his research group.

For financial suport I am indebted to the Academy of Finland, to the Finnish Cultural Foundation and to the Jenny and Antti Wihuri Foundation. 


\section{Contents}

Introduction

1. Closed s-games

2. An approximation theorem for closed s-games $\quad 7$

3. The languages $L_{\lambda \kappa}$ and $\lambda \kappa$-Vaught sentences 9

4. On determinacy 13

5. Applications of games to the model theory of infinitary languages $\quad 19$

References $\quad 31$ 


\section{Introduction}

In this doctoral thesis we study the model theory of the languages $L_{\lambda \kappa}$, $M_{\lambda \kappa}$ and $\lambda \kappa$-Vaught sentences. The languages $L_{\lambda \kappa}$ were first introduced by $\mathrm{A}$. Tarski and since then they have been studied in $[\mathrm{Ka}]$ by $\mathrm{C}$. Karp, in [Ke] by $\mathrm{H}$. J. Keisler and in $[\mathrm{Ba}]$ by K. J. Barwise, among others. $\omega_{1} \omega$-Vaught sentences were first introduced by R. L. Vaught in [Va]. Earlier, L. Svenonius had studied a special case of $\omega_{1} \omega$-Vaught sentences in [Sv]. Since then $\lambda \kappa$-Vaught sentences have been studied in [KM] by M. Karttunen and in [HM] and [Ma2] by V. Harnik and M. Makkai, e.g. (In [KM] Karttunen actually studies Hintikka-Rantala [HR] languages $N_{\lambda \kappa}$ with maximal semantics instead of $\lambda \kappa$-Vaught sentences, but these are essentially the same.) $M_{\lambda \kappa}$ languages were first defined by $M$. Karttunen in [KM] by modifying the definition of $N_{\lambda \kappa}$-languages (see above).

The need to study infinitary languages arose when the expressive power of finitary languages proved to be rather limited. For example we cannot express even in $L_{\infty w}$ that an ordering is wellfounded.

For a long time, there have been a lot of results on languages that allow only relatively little quantifying, like $L_{\omega \omega}$ or $L_{\omega_{1} \omega}$, but very little has been known of languages that allow a lot of quantifying, like $L_{\kappa+\kappa}, \kappa>\omega$. In this doctoral thesis the author has tried to answer the most obvious questions on the expressive power of the languages $L_{\lambda \kappa}, M_{\lambda \kappa}$ and the $\lambda \kappa$-Vaught sentences. The results are new, of course, but the main results in Chapter 5 have been known in the case $\kappa=\omega$ (see [Ma1]). This work continues the work started in [Oi2], [Oi3] and [Oi4]. The reader can find in $[\mathrm{Ke}],[\mathrm{Di}]$ and $[\mathrm{KM}]$, e.g., what is known about such properties of the languages $L_{\lambda \kappa}, M_{\lambda \kappa}$ and the $\lambda \kappa$-Vaught sentences as compactness and downward-Löwenheim-Skolem properties.

The name of this doctoral thesis, "Games and infinitary languages," refers to our project in the University of Helsinki logic seminar led by J. Väänänen and J. Oikkonen. In the project we have tried to use games instead of induction, e.g., in defining concepts and proving theorems in the model theory of infinitary languages (see, e.g., Definition 5.7). In [Ac] P. Aczel has shown that for every inductive definition there is such a game of length $\omega$ that by using the game we can define the same concept as by the induction. J. Oikkonen has shown that in many cases we can let the players play these games more than $\omega$ steps and that in this way we get new natural concepts.

This doctoral thesis is divided into three parts. In the first part, consisting of Chapters 1 and 2, we prove an approximation theorem for the closed s-games. This theorem is the heart of the doctoral thesis. In the second part, consisting of Chapter 4, we take a look at the phenomenon that makes games of length $>\omega$ a lot harder to handle than games of length $\omega$, namely non-determinacy. In the last part, consisting of Chapters 3 and 5, we study the expressive power of the languages $L_{\lambda \kappa}, M_{\lambda \kappa}$ and $\lambda \kappa$-Vaught sentences. For Chapter 5 one does not have to read Chapter 4. 


\section{Closed s-games}

In this doctoral thesis we deal with many different games, but the central technical ideas we use are common to all of them. And so we begin by studying games in general.

We will use the word "game" in the common sense. All the games in this paper are played by two players, which we call $\mathrm{A}$ and $\mathrm{E}$. It is practical to assume that $\mathrm{A}$ is male (he) and $\mathrm{E}$ is female (she) (this convention is from [Ho]). All the moves in these games are made by choosing elements from certain sets. Most of the games in this paper are what we will call standard games:

1.1 Definition. Let $\alpha$ be an ordinal, let $I=\left\{I_{\beta}: \beta<\alpha\right\}$ and $J=\left\{J_{\beta}\right.$ : $\beta<\alpha\}$ be families of sets and let $W \subseteq\left(\Pi_{\beta<\alpha} I_{\beta}\right) \times\left(\Pi_{\beta<\alpha} J_{\beta}\right)$. Then the triple $G=(I, J, W)$ is a standard game (s-game in short) of length $\alpha$. It is played by $A$ and $E$. In every move $\beta<\alpha$, first $A$ chooses an element $x_{\beta} \in I_{\beta}$ and then $E$ chooses an element $y_{\beta} \in J_{\beta}$. We say that $E$ wins the game if the pair of sequences $(x, y)=\left(\left(x_{\beta}\right)_{\beta<\alpha},\left(y_{\beta}\right)_{\beta<\alpha}\right)$ chosen during the game belongs to $W$.

For any cardinals $\kappa$ and $\lambda$ we say that the s-game $G=(I, J, W)$ is a $\lambda, \kappa-$ game if it is of length $\kappa$ and for all $\alpha<\kappa\left|I_{\alpha}\right|,\left|J_{\alpha}\right|<\lambda$. The s-game $G$ is an $\infty, \kappa$-game if for some $\lambda G$ is a $\lambda, \kappa$-game. We define $\lambda, \infty$-games and $\infty, \infty$ games in a similar way.

For every game we associate the concepts "a strategy of A for the game" and "a strategy of $\mathrm{E}$ for the game." In the case of the s-games we do this as follows:

1.2 Definition. A strategy of $A(E)$ for the s-game $G=(I, J, W)$ of length $\alpha$ is a set $F=\left\{f_{\beta}: \beta<\alpha\right\}$ of functions $f_{\beta}: \Pi_{\gamma<\beta} J_{\gamma} \rightarrow I_{\beta}\left(f_{\beta}: \Pi_{\gamma \leq \beta} I_{\gamma} \rightarrow J_{\beta}\right)$.

Usually we are not interested in strategies in general but in winning strategies.

1.3 Definition. A strategy of a player for a game is winning if the player can always win the game by playing according to this strategy. (We say, e.g., that $E$ plays according to her strategy $F=\left\{f_{\beta}: \beta<\alpha\right\}$ for an s-game if in every move $\beta<\alpha$ she chooses $f_{\beta}\left(x_{0}, \ldots, x_{\beta}\right)$, where $x_{0}, \ldots, x_{\beta}$ are the previous choices of $A$.)

Our first major goal in this paper is to prove Theorem 2.3 , which is the main tool in Chapter 5. It does not hold for all s-games, but it does for the closed ones.

1.4 Definition. An s-game $G=(I, J, W)$ of length $\alpha$ is closed if there are such sets $W_{\beta} \subseteq\left(\Pi_{\gamma<\beta} I_{\gamma}\right) \times\left(\Pi_{\gamma<\beta} J_{\gamma}\right), \beta<\alpha$, that

$$
\left(\left(x_{\gamma}\right)_{\gamma<\alpha},\left(y_{\gamma}\right)_{\gamma<\alpha}\right) \in W
$$

if and only if for all $\beta<\alpha$

$$
\left(\left(x_{\gamma}\right)_{\gamma<\beta},\left(y_{\gamma}\right)_{\gamma<\beta}\right) \in W_{\beta} .
$$


We conclude this chapter by defining approximations for the s-games.

We say that a well-founded tree $T=(T,<)$ is neat if for any two different points $x$ and $y$ without immediate predecessor, the sets $\{z \in T: z<x\}$ and $\{z \in T: z<y\}$ are not the same. For any cardinals $\kappa$ and $\lambda$ we say that $T=(T,<)$ is a $\lambda, \kappa$-tree if it is a neat, well-founded tree and no point in $T$ has $\geq \lambda$ immediate successors and there are no branches of length $\geq \kappa$ in $T$. As with the games, we say that $T$ is an $\infty, \kappa$-tree if it is a $\lambda, \kappa$-tree for some $\lambda$. Similarly we define $\lambda, \infty$-trees and $\infty, \infty$-trees.

1.5 Definition. Let $G=(I, J, W)$ be an s-game of length $\kappa$ for some cardinal $\kappa$ and let $T$ be an $\infty, \kappa$-tree. The $T$-approximation $G^{T}$ of $G$ is the following game played by $A$ and $E$. They play $G^{T}$ as they play $G$ except that during the game they go up the tree $T$ as chosen by $A$. The game is over when they cannot go up the tree any more. So the rules are the following: for each move $\alpha$, first $A$ chooses elements $t_{\alpha} \in T$ and $x_{\alpha} \in I_{\alpha}$ so that

1. if $\alpha$ is a successor, $\alpha=\beta+1$, then $t_{\alpha}$ is an immediate successor of $t_{\beta}$;

2. if $\alpha$ is a limit, then $t_{\alpha}=\sup _{\beta<\alpha} t_{\beta}$.

After this, the player $E$ chooses $y_{\alpha} \in J_{\alpha}$. The game continues only as long as $A$ can choose $t_{\alpha}$ satisfying 1 and 2 . When the game is over, the players have made $\alpha$ moves for some $\alpha<\kappa$ and they have chosen sequences $x=\left(x_{\beta}\right)_{\beta<\alpha}, y=\left(y_{\beta}\right)_{\beta<\alpha}$ and $\left(t_{\beta}\right)_{\beta<\alpha}$. E has won if $\left(\left(x_{\beta}\right)_{\beta<\gamma},\left(y_{\beta}\right)_{\beta<\gamma}\right) \in W_{\gamma}$ for all $\gamma \leq \alpha$.

1.6 Definition. A strategy $F$ of $A(E)$ for the game $G^{T}$ is a set $F=\left\{f_{\alpha}\right.$ : $\alpha<\kappa\}$ of functions $f_{\alpha}: \Pi_{\beta<\alpha} J_{\beta} \rightarrow T \times I_{\alpha}\left(f_{\alpha}: \Pi_{\beta \leq \alpha} T \times I_{\beta} \rightarrow J_{\alpha}\right)$.

Let $T$ and $T^{\prime}$ be ordered sets. We say that a function $g: T \rightarrow T^{\prime}$ is order preserving if for any $x, y \in T, g(x)<g(y)$, if $x<y$.

1.7 Lemma. Let $G$ be an s-game of length $\kappa$.

1. If $E$ has a winning strategy for $G$, then for all $\infty, \kappa$-trees $T$ she has a winning strategy for $G^{T}$, too.

2. Let $T$ and $T^{\prime}$ be $\infty, \kappa$-trees. If there is an order-preserving function $g: T \rightarrow$ $T^{\prime}$ and $E$ has a winning strategy for $G^{T^{\prime}}$, then she has a winning strategy for $G^{T}$, too.

The lemma follows immediately from the definitions.

\section{An approximation theorem for closed s-games}

We begin this chapter by proving a combinatorial lemma, which is due to $D$. Kurepa [KD] (see [To]), as A. Levy pointed out to the author.

Let $\lambda$ and $\kappa$ be infinite cardinals and let $T$ be a $\lambda, \kappa$-tree. We put $T^{\prime}$ to be the set of all totally ordered subsets $S$ of $T$ which are closed downward (i.e. $y \in S$ whenever $x \in S$ and $y<x$ ). We order $T^{\prime}$ by inclusion. Then we can easily see that

(*) $\quad T^{\prime}$ is a $\lambda, \kappa-$ tree. 
On the other hand $T^{\prime}$ is essentially larger than $T$ :

2.1 Lemma. There is no order-preserving function

$$
g: T^{\prime} \rightarrow T
$$

Proof. Assume there is such $g: T^{\prime} \rightarrow T$. We define for all $\alpha<\kappa$

$$
x_{\alpha}=g\left(\left\{y \in T: \exists \beta<\alpha\left(y \leq x_{\beta}\right)\right\}\right) \text {. }
$$

Then $\left(x_{\alpha}\right)_{\alpha<\kappa}$ is an increasing sequence of length $\kappa$ in $T$. This contradicts our assumption that $T$ is a $\lambda, \kappa$-tree. $\square$

2.2 Corollary. Let $T$ be a $\lambda, \infty$-tree. We assume that for any $\lambda, \kappa$-tree $U$ there is an order-preserving function $g: U \rightarrow T$. Then there is a branch of length $\kappa$ in $T$.

Proof. The corollary follows immediately from Lemma 2.1 and (*).

2.3 The Approximation Theorem. Let $\lambda$ and $\kappa$ be infinite cardinals, let $G=(I, J, W)$ be a closed $\lambda, \kappa$-game and let $\mu$ satisfy the condition below. If for all $\mu, \kappa$-trees $U$ the player $E$ has a winning strategy for $G^{U}$, then she has a winning strategy for $G$, too.

The condition for $\mu$ is the following: If $\lambda$ is a successor or $\operatorname{cf}(\lambda) \geq \kappa$ then

$$
\mu=\bigcup\left\{\left(2^{\left(\beta^{\gamma}\right)}\right)^{+}: \beta, \gamma \text { cardinals and } \beta<\lambda, \gamma<\kappa\right\}
$$

and otherwise

$$
\mu=\bigcup\left\{\left(2^{\left(\lambda^{\gamma}\right)}\right)^{+}: \gamma \text { cardinal and } \gamma<\kappa\right\} .
$$

Proof. We begin this proof by defining $\alpha$-strategies, which will be the link between Corollary 2.2 and this theorem.

2.4 Definition. Let $\alpha<\kappa$ be an ordinal. An $\alpha$-strategy for $G$ is a winning strategy of $E$ for the s-game $G^{\alpha}=\left(I^{\alpha}, J^{\alpha}, W^{\alpha}\right)$, where

$$
\begin{aligned}
& I^{\alpha}=\left\{I_{\beta}: \beta<\alpha\right\} \\
& J^{\alpha}=\left\{J_{\beta}: \beta<\alpha\right\} \\
& W^{\alpha}=\left\{\left(\left(x_{\beta}\right)_{\beta<\alpha},\left(y_{\beta}\right)_{\beta<\alpha}\right) \in\left(\Pi_{\beta<\alpha} I_{\beta}\right) \times\left(\Pi_{\beta<\alpha} J_{\beta}\right):\left(\left(x_{\beta}\right)_{\beta<\gamma},\left(y_{\beta}\right)_{\beta<\gamma} \in\right.\right. \\
& \left.W_{\gamma} \text { for all } \gamma \leq \alpha\right\} .
\end{aligned}
$$

( $I_{\beta}, J_{\beta}$ and $W_{\gamma}$ are as in the definitions of s-game and closedness.)

Let $T$ be the set of all $\alpha$-strategies for $G, \alpha<\kappa$, ordered by inclusion. Then $T$ is a $\mu, \infty$-tree. To prove the theorem it is enough to show that there is a branch of length $\kappa$ in $T$. By Corollary 2.2 it is enough to show that for any $\mu, \kappa$-tree $U$ there is an order-preserving function $g: U \rightarrow T$. To do this we let $\mathrm{U}$ be any $\mu, \kappa$-tree and define $g(u)$ for all $u \in U$. Let $\left\{u_{\beta}: \beta<\alpha\right\}$ be the set 
$\left\{u^{\prime} \in U: u^{\prime}<u\right\}$ enumerated in such a way that $u_{\beta}<u_{\gamma}$ if $\beta<\gamma<\alpha$. By assumption, there is a winning strategy $F=\left\{f_{\beta}: \beta<\kappa\right\}$ of $\mathrm{E}$ for $G^{U}$. We define $g(u)$ to be the following $\alpha$-strategy:

$$
g(u)=\left\{h_{\beta}: \beta<\alpha\right\}
$$

where

$$
h_{\beta}\left(\ldots, x_{\gamma}, \ldots\right)_{\gamma<\beta}=f_{\beta}\left(\ldots,\left(x_{\gamma}, u_{\gamma}\right), \ldots\right)_{\gamma<\beta} .
$$

Clearly this $g$ is order preserving and so there is a branch of length $\kappa$ in $T$. $\square$

In Chapter 5 we will prove several consequences of this theorem.

\section{The languages $L_{\lambda \kappa}$ and $\lambda, \kappa$-Vaught sentences}

In this chapter we prepare for the last two chapters by defining the languages in which we are interested.

Let $\lambda$ and $\kappa$ be cardinals and let $\mu$ be a set of relation-, function- and constant symbols. The set $\mu$ is called the signature. We recall the definition of the language $L_{\lambda \kappa}(\mu)\left(L_{\lambda \kappa}\right.$ in short):

3.1 Definition. If all the relation and function symbols in $\mu$ are of arity $<\kappa$ then the language $L_{\lambda \kappa}(\mu)$ is defined and it is the least class $X$ such that

1. every atomic formula of the signature $\mu$ belongs to $X$;

2. if $\phi \in X$ then $\neg \phi \in X$;

3. if $\Phi$ is a subset of $X$ of cardinality $<\lambda$ and the number of free variables in $\bigwedge \Phi$ (and in $\bigvee \Phi)$ is $<\kappa$ then $\bigwedge \Phi$ and $\bigvee \Phi$ belong to $X$

4. if $\phi \in X$ and $\bar{x}$ is a set of variables of cardinality $<\kappa$ then $\forall \bar{x} \phi$ and $\exists \bar{x} \phi$ belong to $X$.

The semantics of $L_{\lambda \kappa}$ is defined in the usual and obvious way.

The languages $M_{\lambda \kappa}(\mu)\left(M_{\lambda \kappa}\right.$ in short) were first introduced by M. Karttunen in $[\mathrm{KM}]$. Prior to that, J. Hintikka and V. Rantala had introduced in [HR] $N$-languages that were defined by a similar technique. The idea behind these languages is the following. Let $T$ be a syntax tree of some formula $\phi \in L_{\omega \omega}$. Then $T$ has, among others, the following properties:

1. Every node has $<\omega$ immediate successors.

2. Every branch has length $<\omega$.

We can generalize $L_{\omega \omega}$ by increasing the number of immediate successors and the length of branches. The resulting language is $M_{\lambda \kappa}$.

3.2 Definition. If all the relation and function symbols in $\mu$ are of arity $<\kappa$ then $M_{\lambda \kappa}(\mu)$ is defined and a formula of $M_{\lambda \kappa}(\mu)$ is a pair $(T, l)$ where

1. $T$ is a $\lambda, \kappa$-tree;

2. $l$ is a labeling function with the properties: 
a: if $t \in T$ does not have any successors then $l(t)$ is either an atomic or negated atomic formula of the signature $\mu$;

$b$ : if $t \in T$ has exactly one immediate successor then $l(t)$ is of the form $\exists x$ or $\forall x, x$ variable;

c: if $t \in T$ has more than one immediate successor then $l(t)$ is either $\bigvee$ or $\bigwedge$.

To be able to define a semantics for $M_{\lambda \kappa}$, we must define a certain semantic game. Let $A$ be a model (of the signature $\mu$ ) and let $\phi=(T, l)$ be a sentence of $M_{\lambda \kappa}$.

3.3 Definition. The semantic game $S(A, \phi)$ is a game of two players, $A$ and $E$. When the game begins, the players are in the root of $T$ and during the game the players go up the tree $T$. At each move the players are in some node $t \in T$ and it depends on $l(t)$ how they continue the game:

1. If $l(t)=\bigvee(\Lambda)$ then $E(A)$ chooses one immediate successor of $t$ to be the node where the players go next.

2. If $l(t)=\exists x(\forall x)$ then $E(A)$ chooses an element $x^{\mathcal{A}}$ from $\mathcal{A}$ to be an interpretation of $x$. The players go then to the immediate successor of $t$.

3. If $l(t)=\phi(\bar{x})$ then the game is over and $E$ has won if

$$
A \models \phi(\bar{x})\left[\overline{x^{\mathscr{A}}}\right]
$$

The concepts a strategy of A for $S(\mathcal{A}, \phi)$ and a strategy of $\mathrm{E}$ for $S(\mathcal{A}, \phi)$ are defined essentially as in the case of s-games.

Let $\phi$ be a sentence of $M_{\lambda \kappa}$ and $A$ a model.

3.4 Definition. $A \models \phi$ if $E$ has a winning strategy for $S(A, \phi)$.

We list below a couple of properties of these languages that are apparent but still worth noting.

1. For all $\lambda$ and $\kappa L_{\lambda \kappa}$ is a sublanguage of $M_{\lambda \kappa}\left(L_{\lambda \kappa} \leq M_{\lambda \kappa}\right)$, i.e. for any sentence $\phi$ of $L_{\lambda \kappa}$ there is a sentence $\psi$ of $M_{\lambda \kappa}$ which is equivalent to $\phi$.

2. For all $\lambda L_{\lambda \omega}=M_{\lambda \omega}$ i.e. $L_{\lambda \omega} \leq M_{\lambda \omega}$ and $M_{\lambda \omega} \leq L_{\lambda \omega}$.

3. If $\kappa>\omega$ then there is no obvious reason why $M_{\lambda \kappa}$ would be closed under negation. One might think that we could get the negation of $\phi=(T, l)$ by putting $\neg \phi=\sim \phi=\left(T, l^{\prime}\right)$ where $l^{\prime}$ is such that $l^{\prime}(t)=\bigwedge$ if and only if $l(t)=\bigvee$ and so on. But this is not the case because it may happen that for some model $A$ the semantic game $S(A, \phi)$ is non-determined, i.e. neither $\mathrm{E}$ nor $\mathrm{A}$ has a winning strategy. In this case $A \not \models \phi$ and $A \not \neq \sim \phi$. In Chapter 4 we find an example of a non-determined semantic game. The question whether all the languages $M_{\lambda \kappa}$ are closed under negation or not is open to the author.

In Chapter 5 we will need the next two theorems from [KM]. 
3.5 Theorem. Assume $\lambda^{<\kappa}=\lambda$. If $\phi \in M_{\lambda+}(\mu),|\mu| \leq \lambda$, has a model then it has a model of cardinality $\leq \lambda$.

Proof. Simply take some model $A$ of $\phi$ and some element $a \in A$ and close $\{a\}$ under the winning strategy of $\mathrm{E}$ for $S(\mathcal{A}, \phi)$ and under the functions of $\mu$.

3.6 Theorem. $\kappa$ is weakly compact if and only if $M_{\kappa \kappa}$ has the following property: If $\Sigma$ is a set of sentences of $M_{\kappa \kappa},|\Sigma| \leq \kappa$, and every subset of $\Sigma$ of cardinality $<\kappa$ has a model, then $\Sigma$ has a model.

We omit the proof. Theorems 3.5 and 3.6 are true also for the languages $V_{\lambda+}{ }_{\kappa}$ and $V_{\kappa \kappa}$, which will be defined below (see [KM]).

Let $\lambda$ and $\kappa$ be infinite cardinals. The $\lambda \kappa$-Vaught sentences are defined as follows.

3.7 Definition. We assume that all the relation and function symbols in the signature $\mu$ are of arity $<\kappa$. Then a $\lambda \kappa$-Vaught sentence (of the signature $\mu$ ) is a formula of the following form:

$$
\phi=\left(\forall x_{\alpha} \bigwedge_{i_{\alpha} \in I_{\alpha}} \exists y_{\alpha} \bigvee_{j_{\alpha} \in J_{\alpha}}\right)_{\alpha<\kappa} \bigwedge_{\alpha<\kappa} \phi^{i_{0} j_{0} \ldots i_{\alpha} j_{\alpha}}
$$

where $I_{\alpha}$ and $J_{\alpha}, \alpha<\kappa$, are sets of cardinality $<\lambda$ and $\phi^{i_{0} j_{0} \ldots i_{\alpha} j_{\alpha}}, \alpha<\kappa$, are atomic or negated atomic formulas (of the signature $\mu$ ) with the variables from the set $\left\{x_{0}, y_{0}, \ldots, x_{\alpha}, y_{\alpha}\right\}$.

We will write $V_{\lambda \kappa}$ for the language of all $\lambda \kappa$-Vaught sentences. Again, in defining semantics for the language $V_{\lambda \kappa}$, we need a certain semantic game. Let $A$ be a model and $\phi$ a $\lambda \kappa$-Vaught sentence.

3.8 Definition. The semantic game $S(A, \phi)$ is a game of two players, $A$ and $E$. For each move $\alpha<\kappa$, first $A$ chooses an element $x_{\alpha}^{\mathcal{A}}$ from $A$ to be an interpretation for $x_{\alpha}$ and then he chooses some $i_{\alpha} \in I_{\alpha}$. When $A$ has chosen $x_{\alpha}^{A}$ and $i_{\alpha} E$ chooses some $y_{\alpha}^{A}$ from $A$ to be an interpretation for $y_{\alpha}$ and then she chooses some $j_{\alpha} \in J_{\alpha}$. After $\kappa$ moves $E$ wins if

$$
\mathcal{A} \models \phi^{i_{0} j_{0} \ldots i_{\alpha} j_{\alpha}}\left(x_{0}^{\mathcal{A}}, y_{0}^{\mathcal{A}}, \ldots, x_{\alpha}^{\mathcal{A}}, y_{\alpha}^{\mathcal{A}}\right)
$$

for all $\alpha<\kappa$.

There is also another way to define $S(A, \phi)$ : we put

$$
\begin{aligned}
& I=\left\{A \times I_{\alpha}: \alpha<\kappa\right\} \\
& J=\left\{A \times J_{\alpha}: \alpha<\kappa\right\}
\end{aligned}
$$


and define $W \subseteq\left(\Pi_{\alpha<\kappa} \mathcal{A} \times I_{\alpha}\right) \times\left(\Pi_{\alpha<\kappa} \mathcal{A} \times J_{\alpha}\right)$ to be such that

$$
\left(\left(\left(a_{\alpha}, i_{\alpha}\right)\right)_{\alpha<\kappa},\left(\left(b_{\alpha}, j_{\alpha}\right)\right)_{\alpha<\kappa}\right) \in W
$$

if and only if

$$
A \models \phi^{i_{0} j_{0} \ldots i_{\alpha} j_{\alpha}}\left(a_{0}, b_{0}, \ldots, a_{\alpha}, b_{\alpha}\right)
$$

for all $\alpha<\kappa$. Then we can define $S(\mathcal{A}, \phi)$ to be $(I, J, W)$. So $S(\mathcal{A}, \phi)$ is a closed s-game of length $\kappa$.

3.9 Definition. $A \models \phi$ if the player $E$ has a winning strategy for $S(A, \phi)$.

We make the following remarks:

1. In the definition 3.7 we could have let $\phi^{i_{0} j_{0} \ldots i_{\alpha} j_{\alpha}}$ be conjunction or disjunction of $<\lambda$ atomic or negated atomic formulas, e.g., and still get the same language $V_{\lambda \kappa}$.

2. In all cases $M_{\lambda \kappa} \leq V_{\lambda+\kappa}$ and $V_{\lambda \kappa} \leq M_{\lambda \kappa^{+}}$and if $\lambda$ is a successor cardinal or a regular limit cardinal with $\lambda^{<\kappa}=\lambda$ then $M_{\lambda \kappa} \leq V_{\lambda \kappa}$.

3. As in the usual proof of the Gale-Stewart Theorem, we can see that if $\phi \in V_{\lambda \omega}$ then $S(A, \phi)$ is determined, i.e. either $\mathrm{E}$ or $\mathrm{A}$ has a winning strategy. If $\phi \in V_{\lambda \kappa}$ for some $\kappa>\omega$ then $S(\mathcal{A}, \phi)$ does not have to be determined, as we will see in Chapter 4.

4. The languages $V_{\lambda \kappa}$ are not always closed under negation. For example $V_{\omega \omega}(\emptyset)$ is not closed under negation:

$$
\phi=\forall x_{0} \forall x_{1} \ldots \bigwedge_{i<\omega} \wedge\left\{x_{j} \neq x_{i}: j<i\right\}
$$

belongs to $V_{\omega \omega}(\emptyset)$ and $\neg \phi$ does not because $V_{\omega \omega}$ is compact (see the notice after Theorem 3.6). In Corollary 5.12 we have another example of a language $V_{\lambda \kappa}$ that is not closed under negation.

We say that $\phi$ belongs to $V_{\infty \kappa}\left(M_{\infty \kappa}, L_{\infty \kappa}\right)$ if it belongs to $V_{\lambda \kappa}\left(M_{\lambda \kappa}, L_{\lambda \kappa}\right)$ for some $\lambda$. Similarly we define the languages $V_{\lambda \infty}\left(M_{\lambda \infty}, L_{\lambda \infty}\right)$ and $V_{\infty \infty}$ $\left(M_{\infty \infty}, L_{\infty}\right)$.

We conclude this chapter by giving a characterization for $V_{\infty \kappa}$-elementary equivalence.

Let $A$ and $B$ be models and $\alpha$ an ordinal.

3.10 Definition. The Ehrenfeucht-Fraisse game of length $\alpha F_{\alpha}(A, B)$ is a game of two players, $A$ and $E$. For each move $\beta<\alpha$ first $A$ chooses an element $a_{\beta} \in A$ or $b_{\beta} \in B$ and then $E$ chooses an element $b_{\beta} \in B$ if $A$ has chosen $a_{\beta} \in A$, otherwise she chooses $a_{\beta} \in A$. After $\alpha$ moves $E$ wins if the function that takes $a_{\beta}$ to $b_{\beta}$ for all $\beta<\alpha$ is a partial isomorphism. 
As with the semantic game for the $\lambda \kappa$-Vaught sentences, we could find such $I, J$ and $W$ that $F_{\alpha}(A, B)$ is $(I, J, W)$. So $F_{\alpha}(A, B)$ is an s-game of length $\alpha$ and it is closed if all the relations and functions are of arity $<\operatorname{cf}(\alpha)$, as one can immediately see.

Let $\kappa$ be a cardinal and $A$ a model of cardinality $\lambda$.

3.11 Definition. $\phi_{\mathcal{A}}^{\kappa}$ is the following sentence of $V_{\lambda+\kappa}$ :

$$
\phi_{\mathcal{A}}^{\kappa}=\left(\forall x_{\alpha} \bigwedge_{i_{\alpha} \in \mathcal{A}} \exists y_{\alpha} \bigvee_{j_{\alpha} \in \mathcal{A}}\right)_{\alpha<\kappa} \bigwedge_{\alpha<\kappa} \phi^{i_{0} j_{0} \ldots i_{\alpha} j_{\alpha}}\left(x_{0}, y_{0}, \ldots, x_{\alpha}, y_{\alpha}\right)
$$

where $\phi^{i_{0} j_{0} \ldots i_{\alpha} j_{\alpha}}\left(x_{0}, y_{0}, \ldots, x_{\alpha}, y_{\alpha}\right)$ is the conjunction of all atomic or negated atomic formulas $\psi\left(x_{0}, y_{0}, \ldots, x_{\alpha}, y_{\alpha}\right)$ that satisfy

$$
A \models \psi\left(i_{0}, j_{0}, \ldots, i_{\alpha}, j_{\alpha}\right)
$$

3.12 Lemma. (M. Karttunen) If all the relations and functions are of arity $<c f(\kappa)$ then for all models B:

1. E has a winning strategy for $S\left(B, \phi_{A}^{\kappa}\right)$ if and only if she has a winning strategy for $F_{\kappa}(A, B)$;

2. A has a winning strategy for $S\left(B, \phi_{A}^{\kappa}\right)$ if and only if he has a winning strategy for $F_{\kappa}(A, B)$.

Proof. The lemma follows immediately from the fact that when the length of the Ehrenfeucht-Fraisse game $F_{\alpha}(A, B)$ is some limit ordinal, we can require A and $\mathrm{E}$ to choose two elements in every move $\beta<\alpha$, one from $A$ and one from $B$, and that this requirement does not affect the existence of the winning strategies. ㅁ

3.13 Corollary. (M. Karttunen) We assume that $A$ and $B$ are models and all the relations and functions are of arity $<\operatorname{cf}(\kappa)$ Then $A \equiv B\left(V_{\infty \kappa}\right)$ if and only if $E$ has a winning strategy for $F_{\kappa}(A, B)$.

Proof. " $\Leftarrow$ " follows immediately from the definitions. " $\Rightarrow$ " follows from Lemma 3.12. ㅁ

\section{On determinacy}

The languages $M_{\lambda \kappa}$ and $V_{\lambda \kappa}, \kappa>\omega$, are very different from the languages $M_{\lambda \omega}=L_{\lambda \omega}$ and $V_{\lambda \omega}$. One of the main differences is that the games associated with these languages (namely $S(A, \phi)$ and $F_{\alpha}(A, B)$ ) are always determined in the case $\kappa=\omega$ but might be non-determined when $\kappa>\omega$.

Our main goal in this chapter is the following. For all regular cardinals $\kappa$, $\kappa^{<\kappa}=\kappa$, we give an example of two $\kappa^{+}$-like linear orderings $A$ and $B$ such that the Ehrenfeucht-Fraisse game $F_{\alpha}(A, B)$ of length $\alpha$ is non-determined for every $\alpha, \kappa+2 \leq \alpha<\kappa^{+}$. We also give an example of a non-determined sentence. 
4.1 Definition. A game $G$ is determined if either $A$ or $E$ has a winning strategy. Otherwise $G$ is non-determined. A formula $\phi$ of $M_{\infty \infty}$ or $V_{\infty \infty}$ is determined if for all models $A$ the game $S(A, \phi)$ is determined. Otherwise $\phi$ is non-determined.

It is easy to see that all s-games of finite length and all closed s-games of length $\omega$ are determined. (Use the method of the ordinary proof of the GaleStewart Theorem; see, e.g., [Mo].) So the games $F_{\omega}(\mathcal{A}, B), S(\mathcal{A}, \phi)$ and $S(\mathcal{A}, \psi)$ are determined for all models $A$ and $B$ and for all sentences $\phi \in M_{\infty \omega}\left(=L_{\infty \omega}\right)$ and $\psi \in V_{\infty \omega}$.

Our first examples of non-determined games will be straightforward generalizations of the so-called cub-games studied in $[\mathrm{Ku}]$ by $\mathrm{D}$. Kueker.

Throughout this chapter $\kappa$ will be a regular cardinal and $\kappa^{<\kappa}=\kappa$ (we do not assume that $\kappa$ is a limit cardinal). Let $\alpha<\kappa^{+}$and $A \subseteq\left\{x<\kappa^{+}: \operatorname{cf}(x)=\kappa\right\}$.

4.2 Definition. $G_{\alpha}(A)$ is a game of length $\alpha$ played by $A$ and $E$. During the game $A$ and $E$ construct a sequence by choosing elements from $\kappa^{+}$. $E$ aims to play so that the supremums of certain segments of the sequence chosen during the game are in $A$. So the rules of the game are the following. At every move $\beta<\alpha$ first $A$ chooses some element $x_{\beta}<\kappa^{+}$larger than any element chosen earlier in the game and then $E$ chooses some $y_{\beta}<\kappa^{+}$larger than $x_{\beta} . E$ wins if for every $\gamma \leq \alpha, \operatorname{cf}(\gamma)=\kappa$, the supremum of the sequence $\left(y_{\beta}\right)_{\beta<\gamma}$ is in $A$.

These games are not always non-determined as one can easily see, but for some $A$ and $\alpha$ they are.

Let $C \subseteq\left\{x<\kappa^{+}: \operatorname{cf}(x)=\kappa\right\}$. We say that $C$ is $\kappa$-cub if it is unbounded in $\kappa^{+}$and closed under supremums of increasing sequences of length $\kappa$. In $[\mathrm{Ku}]$ the next theorem has been stated in the case $\alpha=\kappa$.

\subsection{Theorem.}

1. If $E$ has a winning strategy for $G_{\alpha}(A)$ for some $\alpha, \kappa \leq \alpha<\kappa^{+}$, then there is a $\kappa$-cub set $C \subseteq A$.

2. If $A$ has a winning strategy for $G_{\alpha}(A)$ for some $\alpha, \kappa \leq \alpha<\kappa^{+}$, then there is a $\kappa$-cub set $C \subseteq\left\{x<\kappa^{+}: \operatorname{cf}(x)=\kappa\right\} \backslash A$.

\section{Proof.}

1. If $\mathrm{E}$ has a winning strategy for $G_{\alpha}(A)$ for some $\alpha, \kappa \leq \alpha<\kappa^{+}$, then she has it for $G_{\kappa}(A)$, too. Let $F=\left\{f_{\alpha}: \alpha<\kappa\right\}$ be this winning strategy. Let $C$ be the set of those $x$ for which $\operatorname{cf}(x)=\kappa$ and $\left\{y<\kappa^{+}: y<x\right\}$ is closed under every $f_{\alpha}, \alpha<\kappa$. Then it is easy to see that $C$ is $\kappa$-cub.

2. Let $\bar{\alpha}$ be the least $\alpha \geq \kappa$ such that A has a winning strategy for $G_{\alpha}(A)$. Let $F=\left\{f_{\alpha}: \alpha<\bar{\alpha}\right\}$ be a winning strategy of $\mathrm{A}$ for $G_{\bar{\alpha}}(A)$. We see immediately that $\bar{\alpha}$ must be a limit ordinal.

Claim: $\operatorname{Cf}(\bar{\alpha})=\kappa$. 
Proof of the claim: To obtain a contradiction we assume that $\operatorname{cf}(\bar{\alpha})=\lambda<\kappa$. Let $\left(\alpha_{i}\right)_{i<\lambda}$ be a cofinal increasing sequence in $\bar{\alpha}$. It is useful to assume that $\alpha_{0}=0$ and that $\left(\alpha_{i}\right)_{i<\lambda}$ is continuous. For every $i<\lambda$ let $\beta_{i}$ be such that $\alpha_{i}+\beta_{i}=\alpha_{i+1}$. We shall define strategies $G_{i}=\left\{g_{k}^{i}: k<\alpha_{i}\right\}$ of $\mathrm{E}$ for $G_{\alpha_{i}}(A)$ so that

1. $G_{i} \subseteq G_{j}$ for all $i<j<\lambda$.

2. If $i<\lambda$ and $\mathrm{E}$ plays according to $G_{i}$ and $\mathrm{A}$ according to $\left\{f_{\alpha}:{ }^{\circ} \alpha<\alpha_{i}\right\}$, then E wins.

The definition goes as follows.

1. If $i=0$ then $G_{i}=\emptyset$.

2. If $i$ is a limit, then $G_{i}=\bigcup_{j<i} G_{j}$.

3. Then consider the case $i=j+1$. Let $x_{k}$ and $y_{k}, k<\alpha_{j}$, be the choices of $\mathrm{A}$ and $\mathrm{E}$ in the game $G_{\alpha_{j}}(A)$ when $\mathrm{A}$ plays according to $\left\{f_{\alpha}: \alpha<\alpha_{j}\right\}$ and E according to $G_{j}=\left\{g_{k}^{j}: k<\alpha_{j}\right\}$. We define a strategy $F^{\prime}$ of $\mathrm{A}$ for $G_{\beta_{i}}(A)$ by putting $F^{\prime}=\left\{f_{k}^{\prime}: k<\beta_{i}\right\}$, where

$$
f_{k}^{\prime}\left(a_{0}, \ldots, a_{n}, \ldots\right)_{n<k}=f_{\alpha_{i}+k}\left(y_{0}, \ldots, y_{m}, \ldots, a_{0}, \ldots, a_{n}, \ldots\right)_{m<\alpha_{j}, n<k} .
$$

Let $G^{\prime}=\left\{g_{k}^{\prime}: k<\beta_{i}\right\}$ be such a strategy of $\mathrm{E}$ that $\mathrm{E}$ wins the game $G_{\beta_{i}}(A)$ if $\mathrm{E}$ plays according to $G^{\prime}$ and $\mathrm{A}$ according to $F^{\prime}$. Now we put $G_{i}=\left\{g_{k}^{i}: k<\alpha_{i}\right\}$, where

$$
g_{k}^{i}\left(x_{0}, \ldots, x_{m}, \ldots\right)_{m<k}=g_{k}^{j}\left(x_{0}, \ldots, x_{m}, \ldots\right)_{m<k},
$$

if $k<\alpha_{j}$ and

$$
g_{k}^{i}\left(x_{0}, \ldots, x_{m}, \ldots\right)_{m<k}=g_{n}^{\prime}\left(x_{\alpha_{j}}, \ldots, x_{m}, \ldots\right)_{\alpha_{j} \leq m<k},
$$

if $k \geq \alpha_{j}$ and $\alpha_{j}+n=k$.

Because in the game $G_{\alpha}(A), \alpha<\kappa^{+}$, only the sequences of length $\kappa$, not the sequences of length $\leq \lambda$, decide which one has won, we see that the strategies $G_{i}, i<\lambda$, satisfy the properties 1 and 2 above. For the same reason $\mathrm{E}$ wins if she plays according to $\bigcup_{i<\lambda} G_{i}$ and $\mathrm{A}$ according to $F$ in the game $G_{\bar{\alpha}}(A)$. But this contradicts the choice of $F$. $\square$ claim.

We show next that there is a $\kappa$-cub set $C \subseteq\left\{x<\kappa^{+}: \operatorname{cf}(x)=\kappa\right\} \backslash A$, which proves the theorem. In principle we do this as in the proof of part 1 of this theorem.

We let $\left(\alpha_{i}\right)_{i<k}$ be a cofinal increasing sequence in $\bar{\alpha}$. As in the proof of the claim we can construct for every $i<\kappa$ and for every $x \in^{\left\{\alpha_{j}: j<i\right\}} \kappa^{+}$a strategy $G_{x}=\left\{g_{\alpha}^{x}: \alpha<\alpha_{i}\right\}$ for $G_{\alpha_{i}}(A)$ with the following properties.

1. If $\mathrm{E}$ plays according to $G_{x}, x \in^{\left\{\alpha_{j}: j<i\right\}} \kappa^{+}$, and A according to $\left\{f_{\alpha}: \alpha<\alpha_{i}\right\}$ then $\mathrm{E}$ wins. 
2. If $x \in\left\{\alpha_{j}: j<i\right\} \kappa^{+}$and $x^{\prime} \in\left\{\alpha_{j}: j<i^{\prime}\right\} \kappa^{+}$and $i<i^{\prime}$ and for every $j \leq i$, $x\left(\alpha_{j}\right)=x^{\prime}\left(\alpha_{j}\right)$ then for every $\alpha<\alpha_{i}, g_{\alpha}^{x}=g_{\alpha}^{x^{\prime}}$.

3. For all $x \in\left\{\alpha_{j}: j<i\right\} \kappa^{+}$and for all $j<i$ the $\alpha_{j}$ :th choice of $G_{x}$ is always $\geq x\left(\alpha_{j}\right)$.

We define

$$
h:\left\{x \in^{\left\{\alpha_{j}: j<i\right\}} \kappa^{+}: i<\kappa\right\} \rightarrow \kappa^{+}
$$

by letting $h(x), x \in\left\{\alpha_{j}: j<i\right\} \kappa^{+}$, be the least element that is greater than all the choices in the game $G_{\alpha_{i}}(A)$ when A plays according to $\left\{f_{\alpha}: \alpha<\alpha_{i}\right\}$ and $\mathrm{E}$ according to $G_{x}$. We define $C$ to be the set of those $c<\kappa^{+}$that satisfy $\operatorname{cf}(c)=\kappa$ and $\left\{y<\kappa^{+}: y<c\right\}$ is closed under $h$ (which, of course, means that if $\operatorname{rng}(x) \subseteq\left\{y<\kappa^{+}: y<c\right\}$ then $\left.h(x)<c\right)$.

Claim: $\mathrm{C}$ is a $k$-cub subset of $\left\{x<\kappa^{+}: \operatorname{cf}(x)=\kappa\right\} \backslash A$.

Proof of the claim: We show only that $C \subseteq\left\{x<\kappa^{+}: \operatorname{cf}(x)=\kappa\right\} \backslash A$ and leave the other part to the reader (recall $\kappa^{<\kappa}=\kappa$ ).

Let $c \in C$ and let $\left(x_{j}\right)_{j<\kappa}$ be a cofinal sequence in $c$. We let $\mathrm{A}$ and $\mathrm{E}$ play the game $G_{\bar{\alpha}}(A)$ so that A plays according to $F$ and $\mathrm{E}$ according to $\bigcup_{i<\kappa} G_{\left(x_{j}\right)_{j<i}}$. Let $y_{i}, i<\bar{\alpha}$, be the choices of $\mathrm{E}$ in this game. The fact that $\mathrm{A}$ uses his winning strategy and the definition of $G_{x}$ implies that $\sup _{i<\bar{\alpha}} y_{i} \notin A$. On the other hand, $\sup _{i<\bar{\alpha}} y_{i}=\sup _{j<\kappa} x_{j}=c$ and so $c \notin A$. व claim.

We say that $A \subseteq\left\{x<\kappa^{+}: \operatorname{cf}(x)=\kappa\right\}$ is $\kappa$-stationary if the intersection of $A$ and any $\kappa$-cub set is non-empty.

4.4 Lemma. There exists $A \subseteq\left\{x<\kappa^{+}: \operatorname{cf}(x)=\kappa\right\}$ satisfying

1. $A$ is $\kappa$-stationary

2. $\left\{x<\kappa^{+}: \operatorname{cf}(x)=\kappa\right\} \backslash A$ is $\kappa$-stationary.

Proof. By the analogous lemma for stationary sets (see, e.g., [Je] Lemma 7.6) there is such a stationary set $A^{\prime} \subseteq \kappa^{+}$that $\kappa^{+} \backslash A^{\prime}$ is also stationary. We put $A=\left\{x \in A^{\prime}: \operatorname{cf}(x)=\kappa\right\}$. We show that $A$ satisfies 1 in the lemma and leave 2 to the reader.

Let $C$ be a $\kappa$-cub set. We define $C^{\prime}$ to be what we get from $C$ by adding to it all the supremums of less than $\kappa$ elements of $C$. Then $C^{\prime}$ is cub and because $A$ is stationary (it is an intersection of a cub set and a stationary set) the intersection of $C^{\prime}$ and $A$ is non-empty. But then the intersection of $A$ and $C$ must also be non-empty. $\square$

4.5 Corollary. Let $A \subseteq\left\{x<\kappa^{+}: \operatorname{cf}(x)=\kappa\right\}$ be as in Lemma 4.4. Then $G_{\alpha}(A)$ is non-determined for all $\alpha, \kappa \leq \alpha<\kappa^{+}$.

Proof. The corollary follows immediately from Theorem 4.3. $\square$

By using this non-determined cub-game we can get an example of a nondetermined Ehrenfeucht-Fraisse game. In this example we will use linear orderings 
$\Phi_{\kappa}(B)$ defined below. They are introduced by J. Conway in order to characterize all $\omega_{1}$-like dense linear orderings (see [NS]).

Let $\eta$ be a saturated dense linear ordering of cardinality $\kappa$ without endpoints (i.e. an $\eta_{\kappa}$-set which exists under the assumptions on $\kappa$ we have made). Let $1+\eta$ be what we get from $\eta$ by adding one element to $\eta$ as the least element of $1+\eta$. For all $B \subseteq\left\{x<\kappa^{+}: \operatorname{cf}(x)=\kappa\right\}$ we define

$$
\Phi_{\kappa}(B)=\bigcup_{\alpha<\kappa^{+}} \tau_{\alpha} \times\{\alpha\}
$$

where $\tau_{\alpha}=1+\eta$, if $\alpha \in B$ and $\tau_{\alpha}=\eta$, if $\alpha \notin B$. We order $\Phi_{\kappa}(B)$ by setting $(x, \alpha)<(y, \beta)$ if $\alpha<\beta$ or $\alpha=\beta$ and $x<y$ (as members of $\tau_{\alpha}$ ). For all $(x, \alpha) \in \Phi_{\kappa}(B)$ we put $r((x, \alpha))=\alpha$.

Let $A \subseteq\left\{x<\kappa^{+}: \operatorname{cf}(x)=\kappa\right\}$ be as in Lemma 4.4. We put $A=\Phi_{\kappa}(A)$ and $B=\Phi_{\kappa}(\emptyset)$.

4.6 Theorem. For all ordinals $\alpha, \kappa+2 \leq \alpha<\kappa^{+}$, the Ehrenfeucht-Fraisse game $F_{\alpha}(A, B)$ is non-determined.

Proof. Let $\sim A=\left\{x<\kappa^{+}: \operatorname{cf}(x)=\kappa\right\} \backslash A$. By Corollary 4.5 and the fact that if $\mathrm{E}$ has a winning strategy for $F_{\alpha}(A, B)$ then she has a winning strategy for $F_{\beta}(A, B)$, for all $\beta \leq \alpha$, it is enough to show:

1. If $\kappa+2 \leq \alpha<\kappa^{+}$and $A$ has a winning strategy for $F_{\alpha}(A, B)$ then he has a winning strategy for $G_{\kappa}(\sim A)$ and

2. If $\mathrm{E}$ has a winning strategy for $F_{\kappa+2}(A, B)$ then she has a winning strategy for $G_{\kappa}(\sim A)$.

We prove only 1 and leave 2 for the reader, because its proof goes like that of 1 . To show 1 we let $\kappa+2 \leq \alpha<\kappa^{+}$and we assume that $\mathrm{A}$ has a winning strategy for $F_{\alpha}(A, B)$. We describe a winning strategy $F=\left\{f_{i}: i<\alpha\right\}$ of $\mathrm{A}$ for $G_{\alpha}(\sim A)$ by playing both $F_{\alpha}(A, B)$ and $G_{\alpha}(\sim A)$ at the same time. We let $A$ and E play $G_{\alpha}(\sim A)$ and $\mathrm{A}^{\prime}$ and E' play $F_{\alpha}(\mathcal{A}, B)$. A' plays according to his winning strategy, A plays according to the strategy we are describing, $\mathrm{E}$ is the opponent of A and plays arbitrarily and E' plays so that she forces A' to play in such a way that from his moves $\mathrm{A}$ can see how to move. We begin.

1. Move $\mathrm{i}=0$ : $\mathrm{A}^{\prime}$ chooses $x_{0}$ from $A$ or $B$ according to his winning strategy. The first move of $\mathrm{A}$ is now given by $f_{0}(\emptyset)=r\left(x_{0}\right)$. Let $\alpha_{0}>f_{0}(\emptyset)$ be the choice of $\mathrm{E}$ and let $\overline{\alpha_{0}}$ be the least such $\alpha>\alpha_{0}$ that $\alpha \notin A$. Now E' chooses first a partial isomorphism

$$
g_{0}:\left\{x \in A: r(x)<\overline{\alpha_{0}}\right\} \rightarrow\left\{x \in B: r(x)<\overline{\alpha_{0}}\right\},
$$

which exists because both of these sets are saturated linear orderings of cardinality $\kappa$ without endpoints. Then $E^{\prime}$ moves by choosing $y_{0}$ according to $g_{0}$, i.e. $y_{0}=g_{0}\left(x_{0}\right)$ or $y_{0}=g_{0}^{-1}\left(x_{0}\right)$. 
2. Move $\mathrm{i}=\mathrm{j}+1$ : A' chooses $x_{i}$ according to his winning strategy. Then A chooses $f_{i}\left(\alpha_{0}, \ldots, \alpha_{j}\right)=\max \left\{r\left(x_{i}\right), \overline{\alpha_{j}}+1\right\}$. As before we let $\alpha_{i}>f_{i}\left(\alpha_{0}, \ldots, \alpha_{j}\right)$ be the choice of $\mathrm{E}$ and let $\overline{\alpha_{i}}$ be the least such $\alpha>\alpha_{i}$ that $\alpha \notin A$. Now E' first chooses such a partial isomorphism

$$
g_{i}:\left\{x \in A: r(x)<\overline{\alpha_{i}}\right\} \rightarrow\left\{x \in B: r(x)<\overline{\alpha_{i}}\right\}
$$

that $g_{j} \subseteq g_{i}$. This $g_{i}$ exists because the sets

$$
\left\{x \in A: \overline{\alpha_{j}} \leq r(x)<\overline{\alpha_{i}}\right\}
$$

and

$$
\left\{x \in B: \overline{\alpha_{j}} \leq r(x)<\overline{\alpha_{i}}\right\}
$$

are saturated linear orderings of cardinality $\kappa$ without endpoints $\left(\overline{\alpha_{j}} \notin \mathcal{A}\right)$. Then E' chooses $y_{i}$ according to $g_{i}$.

3. Move $\mathrm{i}$ limit: if $\sup _{j<i} \alpha_{j}=\sup _{j<i} \overline{\alpha_{j}} \in A$, then $\mathrm{A}$ has won and we are through. Otherwise we continue as in the successor case; the only difference is that now $g_{i}$ extends $\bigcup_{j<i} g_{j}$ which is possible because $\sup _{j<i} \overline{\alpha_{j}} \notin A$.

A must win the game $G_{\alpha}(\sim A)$ because otherwise $\sup _{j<\beta} \overline{\alpha_{j}} \notin A$ for all limit $\beta<\alpha$ and so, by using the strategy described above, E' would win the game $F_{\alpha}(A, B)$ against $A^{\prime}$ even though $A^{\prime}$ uses a winning strategy. Because $\mathrm{E}$ played arbitrarily, the strategy of $\mathrm{A}$ we described above is a winning strategy. $\square$

Because we skipped the proof of 2 , the reader might wonder why the least $\alpha$ for which Theorem 4.6 is true is $\kappa+2$. The reason is the following: if $a_{i} \in A$ and $b_{i} \in B, i<\kappa$, are the choices $\mathrm{A}$ and $\mathrm{E}$ have made during the first $\kappa$ moves in the game and if $\sup _{i<\kappa} a_{i}$ exists but $\sup _{i<\kappa} b_{i}$ does not, then $\mathrm{A}$ can win the game by two moves but not necessarily by one move. Actually it is easy to see that $\mathrm{E}$ has a winning strategy for $F_{\kappa+1}(A, B)$.

By modifying $A$ and $B$ we can construct an example of a non-determined Ehrenfeucht-Fraisse game of length $\alpha$ for all $\kappa+1 \leq \alpha<\kappa^{+}$. We modify $A$ and $B$ by "naming" every increasing sequence that does not have supremum. In doing this the idea is roughly the following: if $a_{i} \in A$ and $b_{i} \in B, i<\kappa$ are the choices $\mathrm{A}$ and $\mathrm{E}$ have made during the first $\kappa$ moves in the game and if $\sup _{i<\kappa} a_{i}$ exists but $\sup _{i<\kappa} b_{i}$ does not, then $\mathrm{A}$ can win the game by one move if he chooses the "name" of the sequence $\left(b_{i}\right)_{i<\kappa}$.

The modifications are the following. We add to the language unary predicate symbols $M(x)$ and $N(x)$ and a binary predicate symbol $R(x, y)$. M will be an ordered set, $N$ the set of "names" and $R$ will connect the name to the sequence. We define $A^{\prime}$ and $B^{\prime}$ so that

1. $\left(M^{\mathcal{A}^{\prime}},<^{\mathcal{A}^{\prime}}\right)=\Phi(A), A$ as in Lemma 4.4 .

2. $\left(M^{B^{\prime}},<^{B^{\prime}}\right)=\Phi(\emptyset)$.

3. For every increasing sequence $\left(x_{i}\right)_{i<\kappa}$ from $M^{A^{\prime}}\left(M^{B^{\prime}}\right)$ for which $\sup _{i<\kappa} x_{i}$ does not exist, there is exactly one $x \in N^{A^{\prime}}\left(x \in N^{B^{\prime}}\right)$ such that $R(x, y)$ if 
and only if $y=x_{i}$ for some $i<\kappa$ and. vice versa, i.e. for every $x \in N^{A^{\prime}}$ $\left(x \in N^{B^{\prime}}\right)$ there is a sequence $\left(x_{i}\right)_{i<\kappa}$ from $M^{\mathcal{A}^{\prime}}\left(M^{B^{\prime}}\right)$ such that $\sup _{i<\kappa} x_{i}$ does not exist and $R(x, y)$ if and only if $y=x_{i}$ for some $i<\kappa$.

4.7 Theorem. $F_{\alpha}\left(A^{\prime}, B^{\prime}\right)$ is non-determined for all $\alpha, \kappa+1 \leq \alpha<\kappa^{+}$.

Proof. This can be proved as Theorem 4.6. Notice that for every $\alpha<\kappa^{+}$ and every partial isomorphism

$$
f:\left\{x \in M^{\alpha^{\prime}}: r(x)<\alpha\right\} \rightarrow\left\{x \in M^{B^{\prime}}: r(x)<\alpha\right\}
$$

there is exactly one partial isomorphism $g$ from

$$
\left\{x \in M^{\mathcal{A}^{\prime}}: r(x)<\alpha\right\} \cup\left\{x \in N^{\mathcal{A}^{\prime}}: \sup \left\{r(y): R^{\mathcal{A}^{\prime}}(x, y)\right\}<\alpha\right\}
$$

to

$$
\left\{x \in M^{B^{\prime}}: r(x)<\alpha\right\} \cup\left\{x \in N^{B^{\prime}}: \sup \left\{r(y): R^{B^{\prime}}(x, y)\right\}<\alpha\right\}
$$

such that $f \subseteq g$. 口

The author considers the next problem the most interesting open one in the model theory of infinitary languages.

4.8 Question. Are the Ehrenfeuch-Fraisse games of length $\kappa$ determined for all cardinals $\kappa$ ? (All the relations and functions are of arity $<c f(\kappa)$.)

We conclude this chapter by giving an example of a non-determined sentence. Let $A$ be as in Theorem 4.6. As in Definition 3.11 we define $\phi \in M_{\kappa^{++} \kappa^{+}}$and $\psi \in V_{\kappa^{++}}$such that for all models $B$ A or $E$ has a winning strategy for $F_{\kappa+2}(A, B)$ if and only if $\mathrm{A}$ or $\mathrm{E}$ has a winning strategy for $S(B, \phi)$ if and only if $\mathrm{A}$ or $\mathrm{E}$ has a winning strategy for $S(B, \psi)$.

4.9 Lemma. $\phi$ and $\psi$ are non-determined. $\square$

\section{Applications of games to the model theory of infinitary languages}

Our first goal in this chapter is an approximation theorem for $\lambda \kappa$-Vaught sentences.

Let

$$
\phi=\left(\forall x_{\alpha} \bigwedge_{i_{\alpha} \in I_{\alpha}} \exists y_{\alpha} \bigvee_{j_{\alpha} \in J_{\alpha}}\right)_{\alpha<\kappa} \bigwedge_{\alpha<\kappa} \phi^{i_{0} j_{0} \ldots i_{\alpha} j_{\alpha}}
$$

be a $\lambda \kappa$-Vaught sentence, $T$ a $\nu, \kappa$-tree and let $\xi=\max \{\lambda, \nu\}$. We define a $T$ approximation $\phi^{T} \in M_{\xi \kappa}$ of $\phi$ by putting $\phi^{T}=\left(T^{\prime}, l\right)$, where $T^{\prime}$ is the $\xi, \kappa$-tree and $l$ is the labeling function defined below. The idea here is to make $\phi^{T}$ be somewhat like the following Vaught sentence

$$
\left(\bigwedge_{t_{\alpha} \in T} \forall x_{\alpha} \bigwedge_{i_{\alpha} \in I_{\alpha}} \exists y_{\alpha} \bigvee_{j_{\alpha} \in J_{\alpha}}\right)_{\alpha<\kappa} \bigwedge_{\alpha<\kappa} \psi^{t_{0} i_{0} j_{0} \ldots t_{\alpha} i_{\alpha} j_{\alpha}}
$$


where $\psi^{t_{0} i_{0} j_{0} \ldots t_{\alpha} i_{\alpha} j_{\alpha}}$ is $\phi^{i_{0} j_{0} \ldots i_{\alpha} j_{\alpha}}$ if $\left(t_{0}, \ldots, t_{\alpha}\right)$ form an increasing sequence in $T$ and otherwise it is $\forall x(x=x)$.

The set of elements of $T^{\prime}$ will be the set of all sequences of the form

$$
\left(t_{\beta}, \forall, i_{\beta}, \exists, j_{\beta}\right)_{\beta<\alpha}
$$

or

$$
\left(t_{\beta}, \forall, i_{\beta}, \exists, j_{\beta}\right)_{\beta<\alpha} \frown\left(t_{\alpha}\right)
$$

or

$$
\left(t_{\beta}, \forall, i_{\beta}, \exists, j_{\beta}\right)_{\beta<\alpha} \frown\left(t_{\alpha}, \forall\right)
$$

or

$$
\left(t_{\beta}, \forall, i_{\beta}, \exists, j_{\beta}\right)_{\beta<\alpha} \frown\left(t_{\alpha}, \forall, i_{\alpha}\right)
$$

or

$$
\left(t_{\beta}, \forall, i_{\beta}, \exists, j_{\beta}\right)_{\beta<\alpha} \frown\left(t_{\alpha}, \forall, i_{\alpha}, \exists\right)
$$

where $\alpha<\kappa$ and for all $\beta \leq \alpha t_{\beta} \in T, i_{\beta} \in I_{\beta}$ and $j_{\beta} \in J_{\beta}$. Furthermore, $t_{\beta+1}$ is always an immediate successor of $t_{\beta}$ and if $\beta$ is a limit ordinal then $t_{\beta}$ is the supremum of $\left\{t_{\gamma}: \gamma<\beta\right\}$. We order $T^{\prime}$ by the initial segment relation.

For all $x \in T^{\prime}$ we define $l(x)$ as follows.

1. If $x=\left(t_{\beta}, \forall, i_{b}, \exists, j_{\beta}\right)_{\beta<\alpha}$ and $x$ does not have any successors then $l(x)=$ $\bigwedge_{\beta<\alpha} \phi^{i_{0} j_{0} \ldots i_{\beta} j_{\beta}}$.

2. If $x=\left(t_{\beta}, \forall, i_{\beta}, \exists, j_{\beta}\right)_{\beta<\alpha}$ and $x$ has a successor then $l(x)=\bigwedge$.

3. If $x=\left(t_{\beta}, \forall, i_{\beta}, \exists, j_{\beta}\right)_{\beta<\alpha} \frown\left(t_{\alpha}\right)$ then $l(x)=\forall$.

4. If $x=\left(t_{\beta}, \forall, i_{\beta}, \exists, j_{\beta}\right)_{\beta<\alpha} \frown\left(t_{\alpha}, \forall\right)$ then $l(x)=\bigwedge$.

5. If $x=\left(t_{\beta}, \forall, i_{\beta}, \exists, j_{\beta}\right)_{\beta<\alpha} \frown\left(t_{\alpha}, \forall, i_{\alpha}\right)$ then $l(x)=\exists$.

6. If $x=\left(t_{\beta}, \forall, i_{\beta}, \exists, j_{\beta}\right)_{\beta<\alpha} \frown\left(t_{\alpha}, \forall, i_{\alpha}, \exists\right)$ then $l(x)=\bigvee$.

The next lemma follows immediately from the definitions.

5.1 Lemma. For all models $A$ :

1. E has a winning strategy for $[S(A, \phi)]^{T}$ if and only if she has a winning strategy for $S\left(A, \phi^{T}\right)$ (i.e. $\left.A \models \phi^{T}\right)$;

2. A has a winning strategy for $[S(A, \phi)]^{T}$ if and only if he has a winning strategy for $S\left(A, \phi^{T}\right)$.

Vaught himself has defined approximations $\phi^{\alpha}$ for all $\omega_{1} \omega$-Vaught sentences $\phi$, see [Mal] (or [Va]). One might ask how different the approximations defined here are from those defined by Vaught. The next lemma answers this. We skip the easy proof.

5.2 Lemma. Let $\phi$ be an $\omega_{1} \omega$-Vaught sentence.

1. For every ordinal $\alpha$ there is an $\infty, \omega$-tree $T$ such that $\models \phi^{T} \leftrightarrow \phi^{\alpha}$.

2. For every $\infty, \omega$-tree $T$ there is an ordinal $\alpha$ such that $\models \phi^{\alpha} \rightarrow \phi^{T}$. 
We immediately get the next corollary from Lemmas 5.1 and 1.7. Then

5.3 Corollary. Let $\phi$ be an $\infty \kappa$-Vaught sentence and $T$ and $T^{\prime} \infty, \kappa$-trees.

1. $=\phi \rightarrow \phi^{T}$.

2. If there is an order preserving function $g: T \rightarrow T^{\prime}$ then $\models \phi^{T^{\prime}} \rightarrow \phi^{T}$.

The next theorem follows immediately from Lemma 5.1 and Theorem 2.3. In the case $\kappa=\omega$ it is due to R. L. Vaught ([Va]).

5.4 Approximation Theorem. If $\phi$ is a $\lambda \kappa$-Vaught sentence, $\mathcal{A}$ is of cardinality $<\lambda$ and $\mu$ satisfies the condition below then

$$
\mathcal{A} \models \bigwedge_{T \mu, \kappa-t r e e} \phi^{T} \leftrightarrow \phi .
$$

The condition for $\mu$ is the following: if $\lambda$ is a successor or $\operatorname{cf}(\lambda) \geq \kappa$ then

$$
\mu=\bigcup\left\{\left(2^{\left(\beta^{\gamma}\right)}\right)^{+}: \beta, \gamma \text { cardinals and } \beta<\lambda, \gamma<\kappa\right\}
$$

and otherwise

$$
\mu=\bigcup\left\{\left(2^{\left(\lambda^{\gamma}\right)}\right)^{+}: \gamma \text { cardinal and } \gamma<\kappa\right\} .
$$

5.5 Corollary. For all $\infty \kappa$-Vaught sentences $\phi$,

$$
\models \phi \leftrightarrow \bigwedge_{T \infty, \kappa-\text { tree }} \phi^{T} .
$$

5.6 Corollary. For all models $A$ and $B$

$$
A \equiv B\left(M_{\infty \kappa}\right) \text { if and only if } A \equiv B\left(V_{\infty \kappa}\right) \text {. }
$$

We recall from Chapter 3 that $A \equiv B\left(V_{\infty \kappa}\right)$ if and only if $E$ has a winning strategy for $F_{\kappa}(A, B)$.

Our second goal in this chapter is to prove, under certain asumptions on $\kappa$, that one cannot express in $L_{\kappa^{+} \kappa}$ that there is no decreasing sequence of length $\kappa$. This generalizes the undefinability of well-order in $L_{\omega_{1} \omega}$, which is due to M. Morley and E. Lopez-Escobar ([MM] and [Lo]). To do this, we have to be able to construct models for sets of sentences of $L_{\kappa^{+} \kappa}$. For this reason we now present a technique to construct a model out of constants.

Throughout the rest of this chapter, we assume that $\kappa$ is a regular cardinal and $\kappa^{<\kappa}=\kappa$ (again we do not assume that $\kappa$ is a limit cardinal). We also assume that the signature $\mu$ is of cardinality $\leq \kappa$ and that all the relation and function symbols in $\mu$ are of arity $<\kappa$. 
Let $\Sigma$ be a set of sentences of $L_{\kappa^{+} \kappa}(\mu),|\Sigma| \leq \kappa$, and let $C=\left\{c_{i}: i<\kappa\right\}$ be a set of new constant symbols. We assume that in every sentence: $\phi \in \Sigma$ all negations are pushed in front of atomic formulas.

Let $\Delta(C) \supseteq \Sigma$ be a fragment of $L_{\kappa^{+}}(\mu \cup C)$ of cardinality $\kappa$, i.e. $\Delta(C)$ is closed under subformulas, under finite operations, under substituting free variables by terms, and $\Delta(C)$ includes all atomic formulas. This $\Delta(C)$ exists under the assumptions on $\kappa$ we have made. Let $\Delta(C)$ be the family of all subsets $S$ of $\Delta(C)$ of cardinality $<\kappa$ that have the property that for all atomic formulas $\phi$ of $L_{\kappa+\kappa}(\mu \cup C)$ either $\phi \notin S$ or $\neg \phi \notin S$.

5.7 Definition. The Hintikka game $H_{\kappa}(\Sigma, \triangleq(C))$ is a game of length $\kappa$ played by $A$ and $E$. During the game $E$ interprets symbols of $\mu$ to the set $C$ to make $C$ a model of $\Sigma$. This is done so that at every move $\alpha<\kappa$ first $A$ asks a question and then $E$ answers the question by choosing some $S_{\alpha} \in \triangleq(C)$. There are eight different ways to form the question:

1. A chooses some $\phi \in \Sigma$; then $E$ must choose $S_{\alpha} \in \underline{\Delta}(C)$ so that $\phi \in S_{\alpha}$.

2. A chooses a closed term $t$; then $E$ must choose $S_{\alpha} \in \underline{\Delta}(C)$ so that $t=t, t=$ $c \in S_{\alpha}$ for some $c \in C$.

3. A chooses $t=t^{\prime} \in \bigcup_{\beta<\alpha} S_{\beta}$, where $t$ and $t^{\prime}$ are closed terms; then $E$ must choose $S_{\alpha} \in \underline{\Delta}(C)$ so that $t^{\prime}=t \in S_{\alpha}$.

4. A chooses $\exists \bar{x} \phi(\bar{x}) \in \bigcup_{\beta<\alpha} S_{\beta}$; then $E$ must choose $S_{\alpha} \in \triangleq(C)$ so that $\phi(\bar{c}) \in S_{\alpha}$ for some $\bar{c} \in C$.

5. A chooses $\forall \bar{x} \phi(\bar{x}) \in \bigcup_{\beta<\alpha} S_{\beta}$ and some sequence $\bar{t}$ of closed terms; then $E$ must choose $S_{\alpha} \in \underline{\Delta}(C)$ so that $\phi(\bar{t}) \in S_{\alpha}$.

6. A chooses $\bigvee \Phi \in \cup_{\beta<\alpha} S_{\beta}$; then $E$ must choose $S_{\alpha} \in \Delta(C)$ so that $\phi \in S_{\alpha}$ for some $\phi \in \Phi$

7. $A$ chooses $\bigwedge \Phi \in \bigcup_{\beta<\alpha} S_{\beta}$ and $\phi \in \Phi$; then $E$ must choose $S_{\alpha} \in \triangleq(C)$ so that $\phi \in S_{\alpha}$.

8. A chooses $t=t^{\prime}$ and $\phi(t)$ from $\bigcup_{\beta<\alpha} S_{\beta}$, where $t$ and $t^{\prime}$ are closed terms; then $E$ must choose $S_{\alpha} \in \underline{\Delta}(C)$ so that $\phi\left(t^{\prime}\right) \in S_{\alpha}$.

$E$ must always choose $S_{\alpha}$ so that $\bigcup_{\beta<\alpha} S_{\beta} \subseteq S_{\alpha}$. A wins if for some $\alpha<\kappa E$ cannot find $S_{\alpha}$ satisfying the rules. Otherwise $E$ wins.

Again we notice that $H_{\kappa}(\Sigma, \underline{\Delta}(C))$ is a closed s-game of length $\kappa$.

Although the definition of the game above is due to J. Oikkonen, we call this game the Hintikka game because it is a generalization of the concept Hintikka set: in the case $\kappa=\omega \mathrm{E}$ has a winning strategy for $H_{\kappa}(\Sigma, \triangleq(C))$ if and only if $\Sigma$ can be extended to a Hintikka set (see [Ma1]).

5.8 Lemma. ( $\kappa$ is a regular cardinal and $\kappa^{<\kappa}=\kappa$ ) Let $\Sigma$ and $\triangleq(C)$ be as above. If $E$ has a winning strategy for $H_{\kappa}(\Sigma, \triangleq(C))$ then $\Sigma$ has a canonical model $A$, i.e. for every $a \in A$ there is $c \in C$ with $c^{A}=a$. 
Proof. We assume that $\mathrm{E}$ has a winning strategy for $H_{\kappa}(\Sigma, \underline{\Delta}(C))$. We let A and $\mathrm{E}$ play the game $H_{\kappa}(\Sigma, \underline{\Delta}(C))$ so that $\mathrm{E}$ plays according to her winning strategy and $\mathrm{A}$ as described below. The idea here is to make $\mathrm{A}$ ask all the possible questions he can. For this let $g: \kappa \rightarrow \kappa \times \kappa$ be one-one and onto with the property that if $g(x)=(y, z)$ then $y \leq x$. In each move $\alpha<\kappa$ we let $Q_{\alpha}$ be the set of all possible questions $A$ can ask in that move. This set depends on how the players have played earlier. In any case $\left|Q_{\alpha}\right| \leq \kappa$ because $\kappa^{<\kappa}=\kappa$ and we can enumerate it as $Q_{\alpha}=\left\{q_{\beta}^{\alpha}: \beta<\kappa\right\}$. The question A chooses in move $\alpha$ is $q_{\beta}^{\gamma}$ if $g(\alpha)=(\gamma, \beta)$.

Let $\left\{S_{\alpha}: \alpha<\kappa\right\}$ be the set of answers of $\mathrm{E}$ in the game where $\mathrm{E}$ played according to her winning strategy and A according to the strategy described above. We let $S=\bigcup_{\alpha<\kappa} S_{\alpha}$.

In $S$ we have a complete description of $A$. We get the universe of $A$ from $C$ as follows. In $C$ we define an equivalence relation $\sim$ by $c \sim c^{\prime}$ if $c=c^{\prime} \in S$. Because of 2, 3 and 8 in the definition of the Hintikka game, $\sim$ is an equivalence relation. For all $c \in C$ we write $[c]=\left\{c^{\prime} \in C: c^{\prime} \sim c\right\}$ and define the universe of $\mathcal{A}$ to be $\{[c]: c \in C\}$.

All the symbols of $\mu \cup C$ are interpreted to $A$ in the obvious way. For example if $c \in C$ then $c^{\mathcal{A}}=[c]$ and if $R(x, y)$ is a binary relation symbol then $R^{A}\left([c],\left[c^{\prime}\right]\right)$ if $R\left(c, c^{\prime}\right) \in S$ and so on. It follows immediately from the definition of the Hintikka game that $\mathcal{A}$ is well-defined and a model of $\Sigma$. Trivially $\mathcal{A}$ is canonical. $\square$

We say that $T$ is a wide $\lambda, \kappa$-tree if it satisfies what we require from a $\lambda, \kappa-$ tree except that instead of neatness it is assumed to satisfy only the condition that for every $t \in T$ the set

$$
\left\{t^{\prime} \in T:\left\{u \in T: u<t^{\prime}\right\}=\{u \in T: u<t\}\right\}
$$

is of cardinality $<\lambda$. We use this concept in the next theorem instead of the concept of the $\lambda, \kappa$-tree to make the construction of $T^{*}$ easier. Notice that if for every $\lambda, \kappa$-tree $T$ there is an order-preserving function $g: T \rightarrow U$, then for every wide $\lambda, \kappa$-tree $T^{\prime}$ there is an order-preserving function $g: T^{\prime} \rightarrow U$. This is because every wide $\lambda, \kappa$-tree can be extended to a $\lambda, \kappa$-tree.

5.9 Theorem. ( $\kappa$ is a regular cardinal and $\kappa^{<\kappa}=\kappa$ ) Let $\mu$ be any signature which includes a unary predicate symbol $U$ and a binary predicate symbol $<$. We assume that $\phi$ is a sentence of $L_{\kappa}{ }_{\kappa}(\mu)$ and that for every wide $\left(2^{\kappa}\right)^{+}, \kappa$-tree $T$ there is a model $A$ of $\phi$ and an order-preserving function $g$ : $T \rightarrow\left(U^{A},<^{A}\right)$. Then there is a model $A$ of $\phi$ such that $\left(U^{A},<^{A}\right)$ contains an increasing sequence of length $\kappa$.

Proof. Let $D=\left\{d_{\alpha}: \alpha<\kappa\right\}$ be a set of new constants. To prove the theorem it is enough to show that the set

$$
\Sigma=\{\phi\} \cup\left\{d_{i}<d_{j}: i<j<\kappa\right\} \cup\left\{U\left(d_{i}\right): i<\kappa\right\}
$$


has a model. Let $C=\left\{c_{\alpha}: \alpha<\kappa\right\}$ be again a set of new constants and let $\Delta(C)$ and $\Delta(C)$ be as in the definition of the Hintikka game (now the signature is $\mu \cup D)$. By Lemma 5.8 it is enough to show that $\mathrm{E}$ has a winning strategy for $H_{\kappa}(\Sigma, \Delta(C))$. By the Approximation Theorem 2.3 it is enough to show that $\mathrm{E}$ has a winning strategy for $\left[H_{\kappa}(\Sigma, \Delta(C))\right]^{T}$ for every $\left(2^{\kappa}\right)^{+}, \kappa$-tree $T$.

We show this. Let $T$ be an arbitrary $\left(2^{\kappa}\right)^{+}, \kappa$-tree. The idea here is the following. We define a model $A$ of $\phi$ so that $\mathrm{E}$ can play the game $\left[H_{\kappa}(\Sigma, \Delta(C))\right]^{T}$ by putting to $S_{\alpha}$ only "what is true in $A$." If she can do this, she must win. The only problem she must face in doing this is that A can make her interpret a lot of new constants $d_{\alpha}$ to $A$ so that in all cases $d_{\alpha}^{A}<^{A} d_{\alpha^{\prime}}^{A}$ if $\alpha<\alpha^{\prime}$. So we must make $\left(U^{A},<^{A}\right)$ a very rich ordering.

We put

$$
T^{*}=\left\{(t, N, n): t \in T, N \in^{\{u \in T: u \leq t\}} \kappa, n \leq N(t)\right\} .
$$

We define an ordering of $T^{*}$ by putting $(t, N, n)<\left(t^{\prime}, N^{\prime}, n^{\prime}\right)$ if and only if

1. $t<t^{\prime}$ and $N(x)=N^{\prime}(x)$ for all $x \leq t$

or

2. $t=t^{\prime}$ and $N=N^{\prime}$ and $n<n^{\prime}$.

Because $\kappa$ is regular $T^{*}$ is a wide $\left(2^{\kappa}\right)^{+}, \kappa$-tree.

Let $A$ be such a model of $\phi$ that there is an order-preserving function $g$ : $T^{*} \rightarrow\left(U^{\mathcal{A}},<^{\mathcal{A}}\right)$. By using $\mathcal{A}$ we can now describe a winning strategy of $\mathrm{E}$ for $\left[H_{\kappa}(\Sigma, \underline{\Delta}(C))\right]^{T}$.

We assume that $\mathrm{A}$ and $\mathrm{E}$ have played $\alpha$ moves. A has chosen the elements $\left\{t_{\beta}: \beta<\alpha\right\}$ from $T$ and asked the questions $\left\{q_{\beta}: \beta<\alpha\right\}$. E has answered with the sets $\left\{S_{\beta}: \beta<\alpha\right\}$. For some $i, j<\kappa$, E has interpreted all $c_{\beta}, \beta<i$, and $d_{\beta}, \beta<j$, to $A$ and nothing else. We write $c_{\beta}^{A}$ and $d_{\beta}^{A}$ for these interpretations. $\mathrm{E}$ has done this so that if some constant $c \in C$ or $d \in D$ exists in some sentence $\phi$ in $\bigcup_{\beta<\alpha} S_{\beta}$, then $c=c_{\beta}$ or $d=d_{\gamma}$ for some $\beta<i$ or $\gamma<j$. And everything that is in $\bigcup_{\beta<\alpha} S_{\beta}$ is true in $A$ with given interpretations. $E$ has also chosen numbers $N\left(t_{\beta}\right)$ for all $\beta<\alpha$.

On move $\alpha$ A chooses $t_{\alpha} \in T$ and asks a question $q_{\alpha}$. The question $q_{\alpha}$ can be of one of the eight different types in the definition of the Hintikka game. We assume that $q_{\alpha}$ is of type 2 and describe how $\mathrm{E}$ answers. In all other cases $\mathrm{E}$ can answer similarly by keeping in mind that everything in $\bigcup_{\beta<\alpha} S_{\beta}$ is true in $A$ with the given interpretations.

So A has chosen a closed term $t$. Let $i^{\prime}<\kappa$ and $j^{\prime}<\kappa$ be such that $i^{\prime}>i$, $j^{\prime}>j$ and if $c \in C$ or $d \in D$ exists in $t$ then $c=c_{\beta}$ or $d=d_{\gamma}$ for some $\beta<i^{\prime}$ or $\gamma<j^{\prime}$. Then $\mathrm{E}$ puts $N\left(t_{\alpha}\right)=j^{\prime}$ and for all $\beta, i \leq \beta<i^{\prime}$, E interprets $c_{\beta}$ arbitrarily and for all $\gamma, j \leq \gamma<j^{\prime}$ E interprets $d_{\gamma}$ to $g\left(\left(t_{\alpha}, N^{\prime}, \gamma\right)\right)$, where $N^{\prime} \in\left\{t_{\beta}: \beta \leq \alpha\right\} \kappa$ and $N^{\prime}\left(t_{\beta}\right)=N\left(t_{\beta}\right)$ for all $\beta \leq \alpha$ (recall that $g$ is an orderpreserving function $\left.g: T^{*} \rightarrow\left(U^{\mathcal{A}},<^{\mathcal{A}}\right)\right)$. By interpreting new constants this way, E can be sure that if $\gamma<\beta$ then $d_{\gamma}^{A}<d_{\beta}^{A}$. 
At this point the interpretation $t^{\mathcal{A}}$ of $t$ is fixed and $\mathrm{E}$ interprets $c_{i^{\prime}}$ to $t^{\mathcal{A}}$. Then she can answer by choosing $S_{\alpha}$ to be

$$
\bigcup_{\beta<\alpha} S_{\beta} \cup\{t=t\} \cup\left\{t=c_{i^{\prime}}\right\} .
$$

By playing as explained above, E wins the game because she puts to $S_{\alpha}$ only what is true in $A$. $\square$

As a corollary of Theorem 5.9, we can prove a generalization of Vaught's Covering Theorem. In the case $\kappa=\omega$ it is due to R. L. Vaught ([Va]) and the proof we will give is analogous to that in [Va].

5.10 Theorem. ( $\kappa$ is a regular cardinal and $\kappa^{<\kappa}=\kappa$ ) Let $\Phi$ be a $\kappa^{+} \kappa-$ Vaught sentence. If $\Phi$ does not have a model, then there is some $\left(2^{\kappa}\right)^{+}, \kappa$-tree $T$ for which $\Phi^{T}$ does not have a model.

Proof. For a contradiction we assume that

$$
\Phi=\left(\forall x_{\alpha} \bigwedge_{i_{\alpha} \in I_{\alpha}} \exists y_{\alpha} \bigvee_{j_{\alpha} \in J_{\alpha}}\right)_{\alpha<\kappa} \bigwedge_{\alpha<\kappa} \phi^{i_{0} j_{0} \ldots i_{\alpha} j_{\alpha}}
$$

is a $\kappa^{+} \kappa$-Vaught sentence of the signature $\mu$ and that for every $\left(2^{\kappa}\right)^{+}, \kappa$-tree $T$ there is a model of $\Phi^{T}$ but there is not a model of $\Phi$. The idea in this proof is to construct a sentence $\Psi$ of $L_{\kappa+\kappa}$ that contradicts Theorem 5.9.

We take two new unary predicate symbols $M(x)$ and $U(x)$, one new binary predicate symbol $<$ and for every $\alpha<\kappa$ and $\left(\ldots, i_{\beta}, j_{\beta}, \ldots\right)_{\beta<\alpha}, i_{\beta} \in I_{\beta}, j_{\beta} \in J_{\beta}$ for all $\beta<\alpha$, we take a new $1+\alpha$-ary predicate symbol $R^{\left(\ldots, i_{\beta}, i_{\beta}, \ldots\right)_{\beta<\alpha}}$.

Let $\Psi$ be the conjunction of $1-6$ below.

1. " $(U,<)$ is a partial ordering and $M$ is closed under the functions of $\mu$ "

2. $\bigwedge\left\{\forall u \forall\left\{\ldots, x_{\beta}, y_{\beta}, \ldots\right\}_{\beta<\alpha}\left(R^{\left(\ldots i_{\beta} j_{\beta} \ldots\right)_{\beta<\alpha}}\left(u, x_{0}, y_{0}, \ldots, x_{\beta}, y_{\beta}, \ldots\right)_{\beta<\alpha} \rightarrow\right.\right.$

$$
\left.\left.\phi^{\left(\ldots i_{\beta} j_{\beta} \ldots\right)_{\beta<\alpha}}\left(x_{0}, y_{0}, \ldots, x_{\beta}, y_{\beta}, \ldots\right)_{\beta<\alpha}\right): i_{\beta} \in I_{\beta}, j_{\beta} \in J_{\beta} \text { for all } \beta<\alpha, \alpha<\kappa\right\}
$$

3. $\forall u \in U\left(R^{\emptyset}(u)\right)$

4. $\bigwedge\left\{\forall u, u^{\prime} \in U \forall\left\{. ., x_{\beta}, y_{\beta}, . .\right\}_{\beta<\alpha}\left(u<u^{\prime} \rightarrow\right.\right.$

$$
\begin{gathered}
\left(R^{\left(. i_{\beta} j_{\beta} . .\right)_{\beta<\alpha}}\left(u, x_{0}, y_{0}, \ldots, x_{\beta}, y_{\beta}, \ldots\right)_{\beta<\alpha} \rightarrow\right. \\
\left.\left.R^{\left(\ldots i_{\beta} j_{\beta} \ldots\right)_{\beta<\alpha}}\left(u^{\prime}, x_{0}, y_{0}, \ldots, x_{\beta}, y_{\beta}, \ldots\right)_{\beta<\alpha}\right)\right) \\
\left.: i_{\beta} \in I_{\beta}, j_{\beta} \in J_{\beta} \text { for all } \beta<\alpha, \alpha<\kappa\right\}
\end{gathered}
$$

5. $\bigwedge\left\{\forall u \forall\left\{\ldots, x_{\beta}, y_{\beta}, \ldots\right\}_{\beta<\alpha}\left(R^{\left(\ldots i_{\beta} j_{\beta} \ldots\right)_{\beta<\alpha}}\left(u, x_{0}, y_{0}, \ldots, x_{\beta}, y_{\beta}, \ldots\right)_{\beta<\alpha} \rightarrow\right.\right.$

$$
\forall u^{\prime} \in U\left(u^{\prime}>u \rightarrow\right.
$$




$$
\begin{gathered}
\left.\left.\forall x_{\alpha} \bigwedge_{i_{\alpha} \in I_{\alpha}} \exists y_{\alpha} \bigvee_{j_{\alpha} \in J_{\alpha}}\left(\neg M\left(x_{\alpha}\right) \vee\left(M\left(y_{\alpha}\right) \wedge R^{\left(i_{0} j_{0} \ldots i_{\alpha} j_{\alpha}\right)}\left(u^{\prime}, x_{0}, y_{0}, \ldots, x_{\alpha}, y_{\alpha}\right)\right)\right)\right)\right) \\
\left.: i_{\beta} \in I_{\beta}, j_{\beta} \in J_{\beta} \text { for all } \beta<\alpha, \alpha<\kappa\right\}
\end{gathered}
$$

6. $\bigwedge\left\{\forall u \forall\left\{\ldots, x_{\beta}, y_{\beta}, \ldots\right\}_{\beta<\alpha}\left(\bigwedge_{\beta<\alpha} R^{\left(\ldots i_{\gamma} j_{\gamma} \ldots\right)_{\gamma<\beta}}\left(u, x_{0}, y_{0}, \ldots, x_{\gamma}, y_{\gamma}, \ldots\right)_{\gamma<\beta} \rightarrow\right.\right.$

$$
\begin{gathered}
R^{\left(\ldots i_{\beta} j_{\beta} \ldots\right)_{\beta<\alpha}}\left(u, x_{0}, y_{0}, \ldots, x_{\beta}, y_{\beta}, \ldots\right)_{\beta<\alpha}: i_{\beta} \in I_{\beta}, j_{\beta} \in J_{\beta} \text { for all } \beta<\alpha, \\
\alpha \text { is a limit ordinal and }<\kappa\}
\end{gathered}
$$

In short $\Psi$ says the following thing. Let $A$ be a model for the signature $\mu \cup\{U, M,<\}$. Then

$$
\mathcal{A} \models \exists\left\{R^{\left(\ldots i_{\beta} j_{\beta} \ldots\right)_{\beta<\alpha}}: i_{\beta} \in I_{\beta}, j_{\beta} \in J_{\beta}, \alpha<\kappa\right\} \Psi
$$

if and only if

$$
" M^{\mathcal{A}} \models \Phi^{\left(U^{\mathcal{A}},<^{\mathcal{A}}\right) " .}
$$

Because for every $\left(2^{\kappa}\right)^{+}, \kappa$-tree $T, \Phi^{T}$ has a model, we see that for every wide $\left(2^{\kappa}\right)^{+}, \kappa$-tree $T^{\prime}$ there are a model $A$ of $\Psi$ and an order preserving $g$ : $T^{\prime} \rightarrow\left(U^{\mathcal{A}},<^{\mathcal{A}}\right)$. Because $\Phi$ does not have a model, there is no model $\mathcal{A}$ where $\left(U^{\mathcal{A}},<^{\mathcal{A}}\right)$ contains an increasing $\kappa$-sequence. Because $\Psi \in L_{\kappa^{+}}$, the existence of $\Psi$ contradicts Theorem 5.9.

As a corollary of Theorem 5.10 , we will prove a generalization of Theorem 5.9. We could also get this corollary from Theorem 5.9 itself by Skolemization. This Skolemization would go somewhat like the proof of Theorem 5.10.

5.11 Corollary. ( $\kappa$ is a regular cardinal and $\kappa^{<\kappa}=\kappa$ ) Let $\mu$ be any signature that includes a unary predicate symbol $U$ and a binary predicate symbol $<$. We assume that $\phi$ is a sentence of $V_{\kappa^{+}}(\mu)$ and that for every $\left(2^{\kappa}\right)^{+}, \kappa$-tree $T$ there is an order-preserving function $g: T \rightarrow\left(U^{\mathcal{A}},<^{\mathcal{A}}\right)$. Then there is a model $A$ of $\phi$ such that $\left(U^{\mathcal{A}},<^{\mathcal{A}}\right)$ consists of an increasing sequence of length $\kappa$.

Proof. To obtain a contradiction let $\phi \in V_{\kappa^{+}}(\mu)$ be such that for every $\left(2^{\kappa}\right)^{+}, \kappa$-tree $T$ there is a model $\mathcal{A}$ of $\phi$ and an order-preserving $g: T \rightarrow\left(U^{\mathcal{A}},<^{\mathcal{A}}\right)$ but there is no model $A$ of $\phi$ such that $\left(U^{A},<^{A}\right)$ consist of an increasing sequence of length $\kappa$.

Let $\psi \in V_{\kappa+\kappa}$ be

$$
\psi=\left(\exists x_{\alpha}\right)_{\alpha<\kappa} \bigwedge_{\beta<\alpha<\kappa} U\left(x_{\beta}\right) \wedge U\left(x_{\alpha}\right) \wedge\left(x_{\beta}<x_{\alpha}\right) .
$$

Then $\phi \wedge \psi$ is a $\kappa^{+} \kappa$-Vaught sentence and it does not have a model. On the other hand, for every $\left(2^{\kappa}\right)^{+} \kappa$-tree $T(\phi \wedge \psi)^{T}$ has a model, because $A \models \psi^{T}$ if and only if there is an order-preserving function $g: T \rightarrow\left(U^{\mathcal{A}},<^{\mathcal{A}}\right)$. This contradicts Theorem 5.10. 口 
5.12 Corollary. ( $\kappa$ is regular cardinal and $\kappa^{<\kappa}=\kappa$ ) Let $\mu$ be any signature which includes a unary predicate symbol $U$ and a binary predicate symbol $<$. Then $V_{\kappa+\kappa}(\mu)$ is not closed under negation.

Proof. Let $\psi \in V_{\kappa^{+}}(\mu)$ be as in the proof of Corollary 5.11. Then $\neg \psi \notin$ $V_{\kappa+\kappa}(\mu)$ by Corollary 5.11. 口

We can also get a sort of separation theorem for $M_{\kappa^{+} \kappa}$ as a corollary of Theorem 5.10. (The method in the proof is analogous to that used in [Va].) For this we need the following theorem. In its most general form it is due to J. Oikkonen. In the case $\kappa=\omega$ it is due to L. Svenonius and R. L. Vaught ([Sv] and [Va]).

We recall that $\Psi$ is $\Sigma_{1}^{1}$ over $L_{\lambda \kappa}(\mu)$ (over $M_{\lambda \kappa}(\mu)$ ) if it is of the form $\exists \bar{S} \psi$, where $\bar{S}$ is a set of relation (and function) symbols and $\psi \in L_{\lambda \kappa}(\mu \cup \bar{S})(\psi \in$ $\left.M_{\lambda \kappa}(\mu \cup \bar{S})\right)$.

5.13 Theorem. ( $\kappa$ is regular cardinal and $\kappa^{<\kappa}=\kappa$ )

1. For all $\Sigma_{1}^{1}$ over $L_{\kappa^{+}{ }_{\kappa}}$ sentences $\exists \bar{S} \psi$ there is a sentence $\phi$ of $V_{\kappa^{+}{ }_{\kappa}}$ such that

(i) $\models \exists \bar{S} \psi \rightarrow \phi$

(ii) for all models $A$ of cardinality $\leq \kappa A \models \phi \rightarrow \exists \bar{S} \psi$.

2. For all sentences $\phi$ of $V_{\kappa^{+}{ }_{\kappa}}$ there is a $\Sigma_{1}^{1}$ over $L_{\kappa+\kappa}$ sentence $\exists \bar{S} \psi$ such that $\models \phi \leftrightarrow \exists \bar{S} \psi$.

We omit the proof of this theorem. In e.g. [Mal] it is proved that if $\exists \bar{S} \psi$ is $\Sigma_{1}^{1}$ over $L_{\omega_{1} \omega}$ then there is a sentence $\phi$ of $V_{\omega_{1} \omega}$ such that for all models $A$ of cardinality $\leq \omega A \models \phi \leftrightarrow \exists \bar{S} \psi$. In that proof $\phi$ is defined so that it is easy to see that $\models \overline{ } \bar{S} \psi \rightarrow \phi$ (E just keeps on embedding $C$ to the model and chooses $\theta_{n} \in \Delta(C)$ according to the model, see [Ma1]; see also the proof of Theorem 5.9 in this doctoral thesis). Essentially the same proof would yield part 1 in Theorem 5.13. This is done in part in [Oi1] but it is doubtful whether the reader can find that paper. Part 2 in Theorem 5.13 can be proved by Skolemization.

Notice that if in the part 1 in Theorem 5.13 the negation of $\exists \bar{S} \psi$ is also $\Sigma_{1}^{1}$ over $L_{\kappa^{+} \kappa}$, then $\models \phi \leftrightarrow \exists \bar{S} \psi$ (use Downward-Löwenheim-Skolem Theorem).

5.14 Separation Theorem for $M_{\kappa+\kappa} \cdot \quad\left(\kappa\right.$ is a regular cardinal and $\kappa^{<\kappa}=$

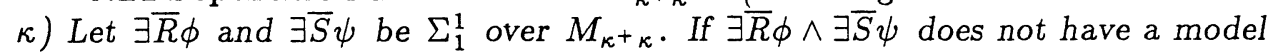
then there is a sentence $\theta \in M_{\left(2^{\kappa}\right)+\kappa}$ such that

$$
\models \exists \bar{R} \phi \rightarrow \theta
$$

and

$$
\vDash \theta \rightarrow \neg \exists \bar{S} \psi \text {. }
$$

Proof. We assume that $\exists \bar{R} \phi \wedge \exists \bar{S} \psi$ does not have a model. By skolemization we can assume that $\exists \bar{R} \phi$ and $\exists \bar{S} \psi$ are $\Sigma_{1}^{1}$ over $L_{\kappa^{+}{ }}$. By Theorem 5.13 there are sentences $\Phi$ and $\Psi$ of $V_{\kappa+\kappa}$ such that 
1. $\models \exists \bar{R} \phi \rightarrow \Phi$ and $\models \exists \bar{S} \psi \rightarrow \Psi$

and

2. for all models $A$ of cardinality $\leq \kappa A \models \Phi \rightarrow \exists \bar{R} \phi$ and $A \models \Psi \rightarrow \exists \bar{S} \psi$.

By the fact that Theorem 3.5 is true also for $V_{\lambda+\kappa}$, as we noticed after Theorem 3.6, we see that $\Phi \wedge \Psi$ does not have a model. By Theorem 5.10 there is a $\left(2^{\kappa}\right)^{+}, \kappa$-tree $T$ such that $(\Phi \wedge \Psi)^{T}$ does not have a model. But then $\Phi^{T} \wedge \Psi^{T}$ does not have a model. Now

$$
\models \Phi^{T} \rightarrow \neg \Psi^{T}
$$

and

$$
\models \neg \Psi^{T} \rightarrow \neg \Psi
$$

and

$$
\models \neg \Psi \rightarrow \neg \exists \bar{S} \psi
$$

and so

$$
\models \Phi^{T} \rightarrow \neg \exists \bar{S} \psi .
$$

On the other hand,

$$
\vDash \exists \bar{R} \phi \rightarrow \Phi
$$

and

$$
\vDash \Phi \rightarrow \Phi^{T}
$$

and so

$$
\models \exists \bar{R} \phi \rightarrow \Phi^{T} \text {. }
$$

5.15 Interpolation Theorem for $L_{\kappa^{+} \kappa} . \quad(\kappa$ is a regular cardinal and $\left.\kappa^{<\kappa}=\kappa\right)$ Let $\phi \in L_{\kappa^{+} \kappa}(\mu)$ and $\psi \in L_{\kappa^{+} \kappa}\left(\mu^{\prime}\right)$. If $\phi \models \psi$ then there is $\theta \in$ $M_{\left(2^{\kappa}\right)+{ }_{\kappa}}\left(\mu \cap \mu^{\prime}\right)$ such that $\phi \models \theta$ and $\theta \models \psi$.

With a refinement of the proof of Theorem 5.13, we can show that in Theorem $5.13 \phi$ can be defined to satisfy the statement: Every relation symbol (excluding identity) which occurs positively (negatively) in $\phi$ occurs positively (negatively) in $\psi$, too. But then in Theorems 5.14 and $5.15 \theta$ satisfies the statement: Every relation symbol (excluding identity) which occurs positively (negatively) in $\theta$ occurs positively (negatively) in $\phi$, too. With our method we cannot get any closer to the Lyndon Interpolation Theorem.

Let

$$
M_{\lambda \kappa}^{n}=\left\{\phi \in M_{\lambda \kappa} \text { : the negation of } \phi \text { belongs to } M_{\lambda \kappa}\right\} \text {. }
$$

We recall that $\Delta\left(L_{\lambda \kappa}\right)\left(\Delta\left(M_{\lambda \kappa}\right)\right)$ is the set of those $\Sigma_{1}^{1}$ over $L_{\lambda \kappa}$ (over $M_{\lambda \kappa}$ ) formulas $\Psi$ for which the negation of $\Psi$ is also $\Sigma_{1}^{1}$ over $L_{\lambda \kappa}$ (over $M_{\lambda \kappa}$ ). 
5.16 Corollary. ( $\kappa$ is a regular cardinal and $\kappa^{<\kappa}=\kappa$ )

$$
\Delta\left(L_{\kappa+\kappa}\right) \quad\left(=\Delta\left(M_{\kappa+\kappa}\right)\right) \subseteq M_{\left(2^{\kappa}\right)+\kappa}^{n} \cdot \text { व }
$$

5.17 Beth's Theorem for $M_{\kappa^{+} \kappa}$. ( $\quad$ is regular cardinal and $\left.\kappa^{<\kappa}=\kappa\right)$ Let $\phi(P) \in M_{\kappa+\kappa}(\mu \cup\{P\})$ and let $\phi\left(P^{\prime}\right)$ be the sentence formed by replacing $P$ everywhere by $P^{\prime}$. We assume that

$$
\phi(P) \wedge \phi\left(P^{\prime}\right) \models \forall \bar{x}\left(P(\bar{x}) \leftrightarrow P^{\prime}(\bar{x})\right) .
$$

Then there is $\theta \in M_{\left(2^{\kappa}\right)+{ }_{\kappa}}(\mu)$ such that

$$
\phi(P) \models \forall \bar{x}(P(\bar{x}) \leftrightarrow \theta(\bar{x})) .
$$

Proof. Let $\bar{c}$ be new constants. Then

$$
(\phi(P) \wedge P(\bar{c})) \wedge\left(\phi\left(P^{\prime}\right) \wedge \neg P^{\prime}(\bar{c})\right)
$$

does not have a model and $\theta(\bar{x})$ will satisfy what we required if $\theta(\bar{c})$ is the separating sentence of these sentences. $\square$

5.18 Separation Theorem for $M_{\kappa \kappa}$. Let $\kappa$ be weakly compact. Let $\exists \bar{R} \phi$ and $\exists \bar{S} \psi$ be $\Sigma_{1}^{1}$ over $M_{\kappa \kappa}$. If $\exists \bar{R} \phi \wedge \exists \bar{S} \psi$ does not have a model, then there is a sentence $\theta \in M_{\kappa \kappa}$ such that

$$
\models \exists \bar{R} \phi \rightarrow \theta
$$

and

$$
\vDash \theta \rightarrow \neg \exists \bar{S} \psi .
$$

Proof. Because $\kappa$ is weakly compact $M_{\kappa \kappa}=\bigcup_{\lambda<\kappa} M_{\lambda+\lambda}$ (this fact is noticed in [KM]). So $\exists \bar{R} \phi$ and $\exists \bar{S} \psi$ are $\Sigma_{1}^{1}$ over $M_{\lambda+\lambda}$ for some $\lambda<\kappa$. Again because $\kappa$ is weakly compact we can choose $\lambda$ to be regular and $\lambda^{<\lambda}=\lambda$. And so Theorem 5.18 follows from Theorem 5.14. $\square$

We could also have got this theorem directly from Theorem 5.13, i.e. to prove Theorem 5.18 we do not need the machinery we have developed in this doctoral thesis; but we do need it for Theorem 5.14. So in the situation of Theorem 5.14, we need more machinery to prove less. It would be interesting to know if we can find $\theta$ from $M_{\kappa+\kappa}$ in Theorem 5.14.

Theorem 5.18 has the following three corollaries.

5.19 Corollary. Let $\kappa$ be weakly compact. Then

$$
\Delta\left(L_{\kappa \kappa}\right) \quad\left(=\Delta\left(M_{\kappa \kappa}\right)\right)=M_{\kappa \kappa}^{n} .
$$

Proof. " $\subseteq$ " follows from Theorem 5.18.

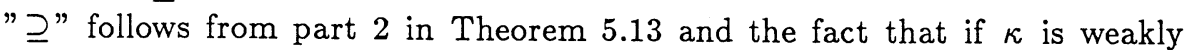
compact then $M_{\kappa \kappa}=\bigcup_{\lambda<\kappa} M_{\lambda+\lambda}$. व 
5.20 Beth's Theorem for $M_{\kappa \kappa}$. Let $\kappa$ be weakly compact. Let $\phi(P) \in$ $M_{\kappa \kappa}(\mu \cup\{P\})$ and let $\phi\left(P^{\prime}\right)$ be the sentence formed by replacing $P$ everywhere by $P^{\prime}$. We assume that

$$
\phi(P) \wedge \phi\left(P^{\prime}\right) \models \forall \bar{x}\left(P(\bar{x}) \leftrightarrow P^{\prime}(\bar{x})\right) .
$$

Then there is $\theta \in M_{\kappa \kappa}(\mu)$ such that

$$
\phi(P) \vDash \forall \bar{x}(P(\bar{x}) \leftrightarrow \theta(\bar{x})) .
$$

Proof. Let $\bar{c}$ be new constants. Then

$$
(\phi(P) \wedge P(\bar{c})) \wedge\left(\phi\left(P^{\prime}\right) \wedge \neg P^{\prime}(\bar{c})\right)
$$

does not have a model and $\theta(\bar{x})$ will satisfy what we required if $\theta(\bar{c})$ is the separant of these sentences. $\square$

We say that theory $T$ in the language $M_{\lambda \kappa}$ is complete if it has the following property: if $\phi \in M_{\lambda \kappa}$ and for some model $A$ of $T A \models \phi$ then $\phi \in T$, i.e. $T$ is a maximal consistent theory in $M_{\lambda \kappa}$.

5.21 Robinson Consistency Theorem for $M_{\kappa \kappa}$. Let $\kappa$ be weakly compact, let $\mu^{\prime}$ and $\mu^{\prime \prime}$ be two signatures and let $\mu$ be $\mu^{\prime} \cap \mu^{\prime \prime}$. Suppose $T$ is a complete theory in $M_{\kappa \kappa}(\mu)$ and $T_{1} \supset T, T_{2} \supset T$ are consistent theories in $M_{\kappa \kappa}\left(\mu^{\prime}\right)$ and $M_{\kappa \kappa}\left(\mu^{\prime \prime}\right)$, respectively. Furthermore suppose $T_{1}$ and $T_{2}$ are of cardinality $\leq \kappa$. Then $T_{1} \cup T_{2}$ is consistent.

Proof. By Theorem 3.6 it is enough to show that for every $\phi \in T_{1}$ and $\psi \in T_{2}$ the set $\{\phi, \psi\}$ is consistent. If it is not, then $\exists \bar{R} \phi \wedge \bar{S} \psi$ does not have a model, $\bar{R}$ is the set of those relation and function symbols in $\phi$ that are not in $\mu$ and $\bar{S}$ is the set of those relation and function symbols in $\psi$ that are not in $\mu$. If $\theta$ is the separant of these sentences, it belongs to $T$ and thus to $T_{2}$, also. But this is impossible because $\{\theta, \psi\}$ is not consistent. $\square$ 


\section{References}

[Ac] Aczel, P.: An introduction to inductive definitions. -In: Handbook of mathematical logic, edited by K. J. Barwise. North-Holland Publishing Company, Amsterdam, London, New York, 1977, pp. 739-782.

[Ba] Barwise, J.: Admissible sets and structures. -Springer-Verlag, Berlin, Heidelberg, New York, 1975.

[Di] Dickmann, M. A.:Large infinitary languages: model theory. -North-Holland Publishing Company, Amsterdam, London, New York, 1975.

[HM] Harnik, V. and Makkai, M.: Applications of Vaught sentences and the covering theorem. -J. Symbolic Logic, vol. 41, 1976, pp. 171-187.

[Ho] Hodges, W.: Building models by games. -Cambridge University Press, Cambridge, London, New York, New Rochelle, Melbourne, Sydney, 1985.

[HR] Hintikka, J. and Rantala, V.: A new approach to infinitary languages. -Ann. Math. Logic, vol. 10, 1976, pp. 95-115.

[Je] Jech, T.: Set theory. -Academic Press, New York, London, 1978.

[Ka] Karp, C.: Languages with expressions of infinite length. -North-Holland Publishing Company, Amsterdam, London, New York, 1964.

[KM] Karttunen, M.: Model theory for infinitely deep languages. -Ann. Acad. Sci. Fenn. Ser. A I Math. Dissertationes, vol. 50, 1984.

[Ke] Keisler, H. J.: Model theory for infinitary logic. -North-Holland Publishing Company, Amsterdam, London, New York, 1971.

[KD] Kurepa, D.: Ensembles ordonnes et ramifies. -Publ. Math. Univ. Belgrade, 4, 1935, pp. 1-138.

[Ku] Kueker, D.: Countable approximations and Lövenheim-Skolem theorems. -Ann. Math. Logic, vol. 11, 1977, pp. 57-103.

[Lo] Lopez-Escobar, E.: On defining well-orderings. -Fund. Math., vol. 59, 1966, pp. 13-21- 299-300.

[Ma1] Makkai, M.: Admissible sets and infinitary logic. -In: Handbook of mathematical logic, edited by K. J. Barwise. North-Holland Publishing Company, Amsterdam, London, New York, 1977, pp. 233-281.

[Ma2] Makkai, M.: Generalizing Vaught sentences from $\omega$ to strong cofinality $\omega$. -Fund. Math., vol. 82, 1974, pp. 105-119.

[MM] Morley, M.:Omitting classes of elements. -In: The theory of models, edited by J. Addison, L. Henkin and A. Tarski. North-Holland Publishing Company, Amsterdam, London, New York, 1965, pp. 265-273.

[Mo] Moschovakis, Y. N.: Descriptive set theory. -North-Holland Publishing Company, Amsterdam, London, New York, 1980.

[NS] Nadel, M. and Stavi, J.: $L_{\infty \lambda}$-equivalence, isomorphism and potential isomorphism. -Trans. Amer. Math. Soc., vol. 236, 1978, pp. 51-74. 
[Oi1] Oikkonen, J.: $\Sigma_{1}^{1}$ over $L_{\kappa^{+} \kappa}$. -In: Vexing Question, An urnful of essays in honour of Veikko Rantala on the occasion of his fiftieth birthday, edited by I. Patoluoto, E. Saarinen and P. Stenman. Reports from the Department of Philosophy University of Helsinki, n:o 3/1983.

[Oi2] Oikkonen, J.: Induction generalized by long games. -Reports of the Department of Mathematics University of Helsinki, March 1985.

[Oi3] Oikkonen, J.: Large infinitary languages and generalized induction. -Reports of the Department of Mathematics University of Helsinki, March 1985.

[Oi4] Oikkonen, J.: The $\Delta$-extension of $L_{\kappa \kappa}$. -Reports of the Department of Mathematics University of Helsinki, August 1985.

[Sv] Svenonius, L.: On the denumerable models of theories with extra predicates. -In: The theory of models, edited by J. W. Addison, L. A. Henkin and A. Tarski. North-Holland Publishing Company, Amsterdam, London, New York, 1965, pp. 376-389.

[To] Todorcevic, S.: Partition relations for partially ordered sets. -Acta Math., vol. $155,1985$.

[Va] Vaught, R. L.: Descriptive set theory in $L_{\omega_{1} \omega}$. -In: Cambridge summer school in mathematical logic, edited by A. R. D. Mathias and H. Rogers. Springer-Verlag Lecture Notes in Mathematics, vol. 337, 1973, pp. 574-598.

University of Helsinki

Department of Mathematics

Hallituskatu 15

00100 Helsinki 10

Finland 